\title{
A general framework for continuum damage models. II. Integration algorithms, with applications to the numerical simulation of porous metals
}

\author{
Francisco Armero a,*, Sergio Oller ${ }^{\mathrm{b}, 1}$ \\ ${ }^{a}$ Structural Engineering, Mechanics and Materials, Department of Civil and Environmental Engineering, University of California, 713 \\ Davis Hall, Berkeley CA 94720, USA \\ ${ }^{\mathrm{b}}$ ETS Ingenieros de Caminos, Canales y Puertos, Universitat Politècnica de Catalunya, Campus Nord, Modulo C1, 08034 Barcelona, \\ Spain
}

Received 21 August 1999; in revised form 20 January 2000

\begin{abstract}
In this paper, we develop numerical algorithms for the integration of the continuum plastic damage models formulated in the general framework identified in Part I of this work. More specifically, we focus our attention on a particular plastic damage model of porous metals, involving a classical von Mises yield criterion coupled with a pressure dependent damage surface to model the nucleation and growth of voids in the metallic matrix. Unilateral damage leading to a sudden change of stiffness in the material's response due to the closing/opening of these voids is also incorporated through the imposition of the unilateral constraint of a positive void fraction, thus, illustrating the clear physical significance added by this framework in the resulting constitutive models. The proposed integration algorithms fully use the modularity of the identified framework, leading in this way to independent integration algorithms for the elastoplastic part and each damage mechanism. Remarkably, all these individual integration schemes share the same formal structure as the classical return mapping algorithms employed in the numerical integration of elastoplastic models, namely an operator split structure consisting of a trial state and the return map imposing the plastic and damage consistency, respectively. A Newton iterative scheme imposes the equilibrium (equal stresses) among the different mechanisms of the response of the material. This modular structure allows to obtain the closed-form consistent linearization, involving in a simple form the algorithmic consistent tangents corresponding to each independent mechanism, thus resulting in a very modular and efficient computational implementation. The performance of the proposed algorithms is illustrated in several representative numerical simulations. () 2000 Elsevier Science Ltd. All rights reserved.
\end{abstract}

Keywords: Return mapping algorithms; Coupled damage plasticity; Porous metals

\footnotetext{
${ }^{*}$ Corresponding author. Fax: +1-510-643-8928.

E-mail address: armero@ce.berkeley.edu (F. Armero).

${ }^{1}$ On leave (Fall semester 1998) in the Department of Civil and Environmental Engineering, UC Berkeley.
} 


\section{Introduction}

In this paper, we develop the numerical integration of continuum plastic damage models formulated in the general framework identified in the work of Armero and Oller (1999) (referred simply as Part I hereafter). More specifically, we investigate the application of the resulting methods to the numerical simulation of the plastic damage response of porous metals. The general framework identified in this work considers a kinematic decomposition of the strains in elastic, plastic and damage parts. The latter is used in the direct and physical modeling of the damage response of the material. A full thermodynamically consistent framework is developed to this purpose. Even though these ideas can be found in early references, usually in the common form of "crack strains" in the modeling of damage in concrete (most notably in the early work of Ortiz (1985) and in the smeared crack models of Bazant and Oh (1983) and Rots et al. (1985), among others), the framework identified in this work not only allows for their extension accommodating plasticity effects in a full thermodynamic context, but also to the identification of a physically motivated unifying framework among other approaches, including the so-called effective stress damage models.

The improved numerical treatment resulting from the considered framework, as developed in detail in this paper, is perhaps even more appealing. This improvement is gained, once more thanks to the modular treatment of the different components of the material's response, from the elastic and plastic parts to the different damage mechanisms that can be present. The modularity in the numerical implementation is achieved by fully maintaining the aforementioned damage strains in the integration schemes. In this context, the response of each mechanism is integrated independently, that is, each damage stress/strain
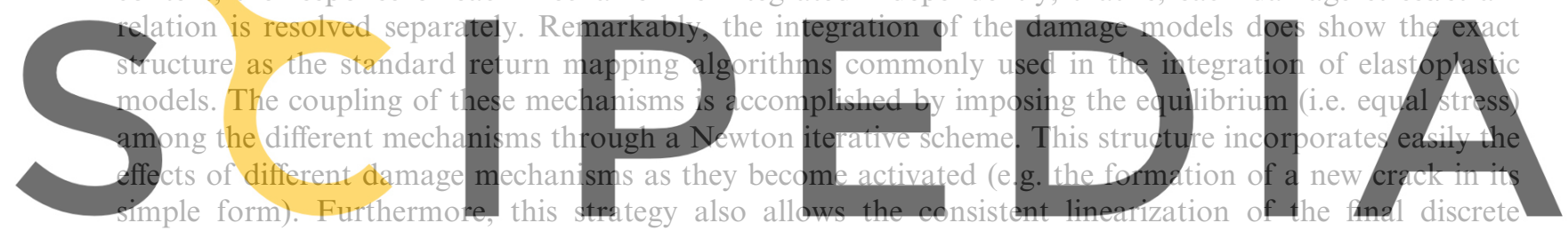

equations, combining in a simple form the algorithmic consistent tangents associated to each component of

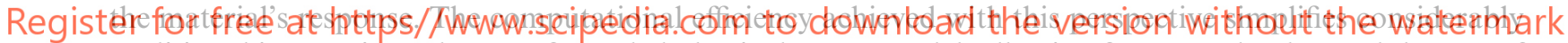
traditional integration schemes of coupled plastic damage model, allowing for example, the modular use of preexisting routines for purely elastoplastic models and routines integrating each damage mechanism. We present complete details of implementation of this novel numerical treatment of the problem, including some representative numerical simulations to illustrate the performance of the resulting numerical algorithms.

Even though we present the development of these new numerical techniques in the general context furnished by the framework developed in Part I, our focus in this paper goes to the formulation and numerical integration of a simple plastic damage model of ductile failure in porous metals, including the effects of closing/opening of voids, an effect usually referred to as unilateral damage or damage deactivation (e.g. Chaboche, 1995; Hansen and Schreyer, 1995). Next, we discuss briefly the physical motivation behind the proposed model.

\subsection{Damage in porous metals: void nucleation and growth}

The study and numerical simulation of the behavior of porous metals is of major importance in industrial applications. Their physically motivated and numerically efficient modeling are some of the principal motivations of this work. The physical mechanisms of interaction between damage and plastic behavior are complicated in nature, as it is their phenomenological representation by means of constitutive modeling. The degradation of the stiffness of the material in damage is due to the fact that, in the course of loading, the effective resisting area diminishes as a result of the generation and expansion of voids and 
microcracks. Damage alone affects the elastic properties of the material, but usually plasticity adds an irreversible component to the inelastic strain evolution. The phenomenon of void nucleation and growth in crystalline metals can be found discussed in detail in many works in the literature; we refer to the comprehensive account given by Atkins and Mai (1985) for a representative example.

There is much evidence on the beginning of failure in bars submitted to tension under room temperature. Normally the failure begins on the natural crystallographic planes, through intergranular fractures or by growth and/or distortion of voids. The problem becomes more complicated at high temperatures because of the appearance of thermally activated creep, changes in the metallographic characteristics, diffusion and also recrystallization of the metallic matrix. The study of these effects falls outside the scope of the simpler models considered herein. For polycrystalline metals under the conditions of interest, the problem can be reduced to three basic types of fracture mechanisms, as explained by Ashby and Tomkins (1980) (Atkins and Mai, 1985, p. 34):

1. Fracture produced by small cracks and voids. This type of phenomenon occurs in the total absence of plasticity and is normally motivated by corrosion and/or abrasion.

2. Fracture effects on small cracks and voids produced by mechanical stresses that exceed the material strength.

Separation of crystallographic planes and fractures throughout the grain boundaries, produced by high stress derived from mechanical actions.

Brittle fracture is normally produced along inter crystallographic planes, while the fracture will be more ductile as distortion mechanisms on the crystal lattice and/or voids or pores are developed.
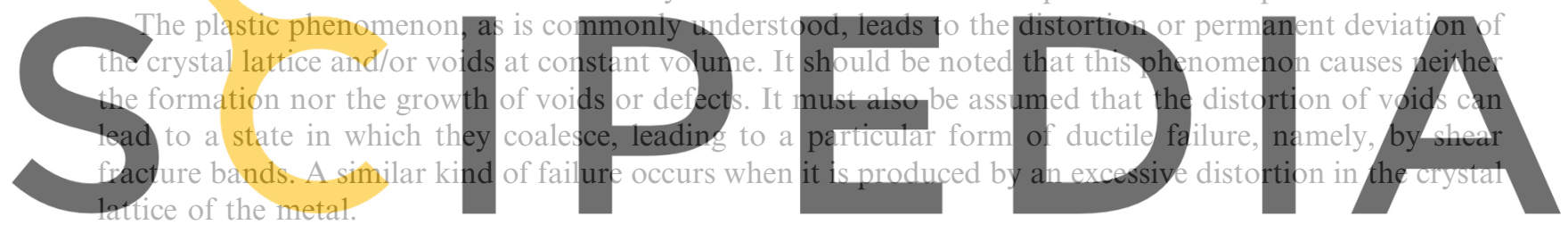

Damage, understood as the nucleation and growth of voids and microcracks, appears then as a

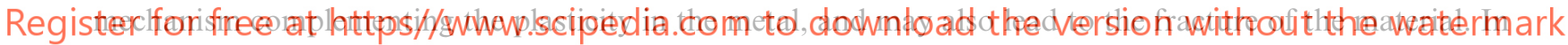

this case, the fracture occurs due to the decreasing effective area as referred to above. In those cases in which there is no creation or nucleation of voids, with the preexisting defects remaining small in size under constant volume, it can be assumed that the mechanical behavior can be described by the simplest, purely deviatoric, von Mises yield surface and the associated plastic evolution equations. This situation implies that the voids can develop permanent distortions only under isochoric states of strain.

A more general plastic model for metal behavior that takes into account void nucleation and growth was proposed by Gurson (1975). Briefly, this formulation incorporates the influence of the hydrostatic pressure within the basic von Mises formulation, allowing for the consideration of void nucleation and growth through the hardening of the material. A direct relation of this hardening mechanism is established with the void fraction in the porous metal. The presence of the second invariant of the deviatoric stresses allows at the same time the modeling of the distortion of the voids and crystal lattice. A similar extension of the classical von Mises criterion incorporating pressure effects due to degradation of the material can be found in the work of Shima and Oyane (1976). We refer to Tvergaard and Needleman (1984) and Agelet and Onate (1989), among many others, for a more complete discussion of these considerations, including numerical simulations based on these models. We point out that these models are simply elastoplastic models, without degrading the elastic stiffness of the material upon unloading. Therefore, their validity should not be expected in (loading/unloading) cycling processes.

In this paper, we propose a broader treatment of the phenomenon of nucleation, growth and distortion of voids in metals through the general continuum plastic damage framework developed in the first part of the paper. In particular, the following features are to be noted: 
(1) The damage part of the model takes into account the nucleation and growth of voids and defects, thus allowing for the appropriate treatment of the concept of decreasing effective area and its influence in the loading/unloading stiffness of the material. The normal to the damage surface governs the creation of defects damaging the material.

(2) The plasticity part of the model is to take care of the distorsion of defects, voids and the crystal lattice, with their orientation established by means of the classical plastic flow. The von Mises yield criterion is considered for this purpose.

In this context, we propose a quadratic damage surface on the hydrostatic pressure and the second invariant of the deviatoric stresses. The need of a combination of both components of the stress in the modeling of the nucleation and growth of voids is well known (Needleman and Rice, 1978; Needleman and Tvergaard, 1984). Physically, the proposed damage surface can be thought in energetic terms as a linear combination of the volumetric and distorsional energies in the material.

Added to these considerations, it is of crucial importance to model the unilateral character of the damage, accounting for the closing/opening of the voids and microcracks in the metal. This feature is easily accomplished in the proposed framework through an unilateral constraint on the damage strains. More specifically, the trace of the damage strains can be related to the void fraction in the metal. In this way, the physically motivated constraint of positive void fraction leads naturally to the modeling of the unilateral damage effects.

An outline of the rest of the paper is as follows: Section 2 develops the new plastic damage model for the modeling of ductile failure of porous metals. To this purpose we first present in Section 2.1 a pressure
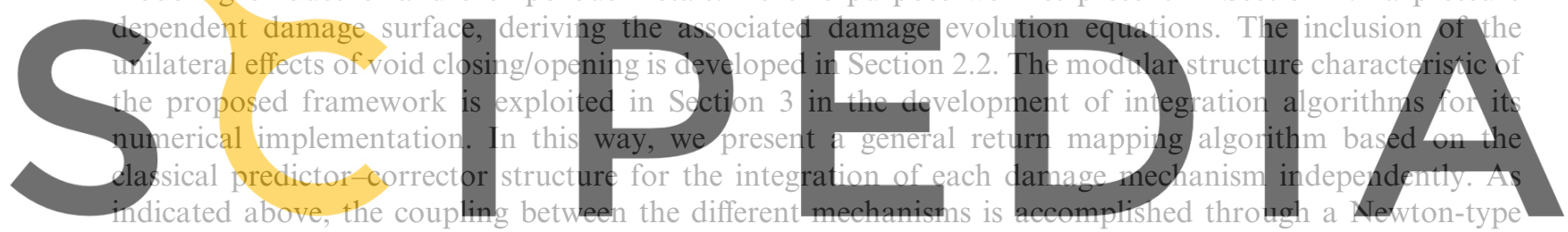

iterative scheme imposing the equilibrium of stresses among them. The particular plastic damage model

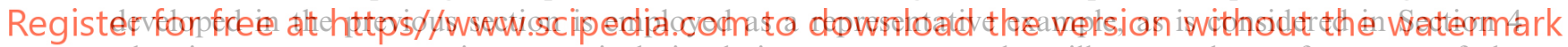
wherein some representative numerical simulations are presented to illustrate the performance of the proposed formulation. Finally, we present in Section 5 a summary of the previous developments together with some additional concluding remarks.

\section{A plastic damage model of failure of metals}

We present in this section a damage model in the framework identified by the generic quadratic damage model presented Part I of this work. Our interest is to illustrate this general framework in the context of plastic damage in metals, characterizing the creation of voids in the resulting porous metal.

In particular, we consider the standard von Mises yield criterion

$$
\phi^{\mathrm{p}}\left(\boldsymbol{\sigma}, q^{\mathrm{p}}\right)=\|\boldsymbol{s}\|-\sqrt{\frac{2}{3}} \underbrace{\left(y_{0}^{\mathrm{p}}-q^{\mathrm{p}}\left(\alpha^{\mathrm{p}}\right)\right.}_{y^{\mathrm{p}}\left(\alpha^{\mathrm{p}}\right)}) \leqslant 0,
$$

for an initial yield limit $y_{0}^{\mathrm{p}}$, the Euclidean norm of the deviatoric stress tensor $s$ such that

$$
\|\boldsymbol{s}\|^{2}=s_{i j} s_{i j} \quad \text { with } \quad \boldsymbol{s}:=\operatorname{dev}[\boldsymbol{\sigma}]=\boldsymbol{\sigma}-\frac{1}{3} p \mathbf{1} \quad \text { and } \quad p=\operatorname{tr}[\boldsymbol{\sigma}] .
$$

Based on this yield condition, the associated plastic evolution equations 


$$
\begin{aligned}
& \boldsymbol{\varepsilon}^{\mathrm{p}}=\gamma^{\mathrm{p}} \boldsymbol{n}_{\phi^{\mathrm{p}}}, \quad \text { where } \boldsymbol{n}_{\phi^{\mathrm{p}}}:=\frac{\partial \phi^{\mathrm{p}}}{\partial \boldsymbol{\sigma}}=\frac{\boldsymbol{s}}{\|\boldsymbol{s}\|}, \\
& \dot{\alpha}^{\mathrm{p}}=\sqrt{\frac{2}{3}} \gamma^{\mathrm{p}},
\end{aligned}
$$

for the plastic strain $\varepsilon^{\mathrm{p}}\left(=\boldsymbol{\varepsilon}-\boldsymbol{\varepsilon}^{\mathrm{e}}-\boldsymbol{\varepsilon}^{\mathrm{d}}\right)$ and the equivalent plastic strain $\alpha^{\mathrm{p}}$, a scalar internal variable modeling the isotropic plastic hardening of the material. The evolution equations (2.3) and (2.4) are complemented with the plastic Kuhn-Tucker loading/unloading conditions

$$
\gamma^{\mathrm{p}} \geqslant 0, \quad \phi^{\mathrm{p}} \leqslant 0, \quad \gamma^{\mathrm{p}} \phi^{\mathrm{p}}=0
$$

and the plastic consistency condition

$$
\gamma^{\mathrm{p}} \dot{\phi}^{\mathrm{p}}=0
$$

for the plastic multiplier $\gamma^{p}$. The numerical simulations presented in Section 4 consider an isotropic linear elastic response, that is, we have the standard relations

$$
\boldsymbol{\sigma}=\mathbf{C}^{\mathrm{e}} \boldsymbol{\varepsilon}^{\mathrm{e}} \quad \text { with } \mathbf{C}^{\mathrm{e}}=\kappa^{\mathrm{e}} \mathbf{1} \otimes \mathbf{1}+2 \mu^{\mathrm{e}}\left[\llbracket-\frac{1}{3} \mathbf{1} \otimes \mathbf{1}\right] \text {, }
$$

for the (constant) elastic bulk modulus $\kappa^{\mathrm{e}}$ and shear modulus $\mu^{\mathrm{e}}$, and the rank four $\square$ and rank two 1 identity tensors.

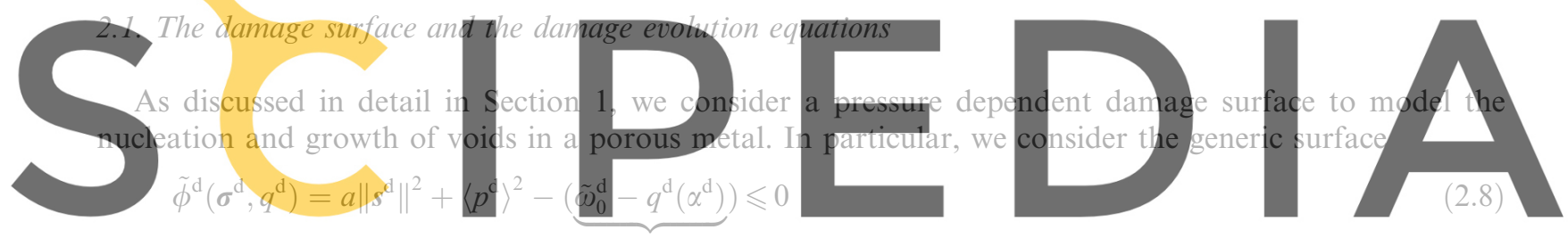

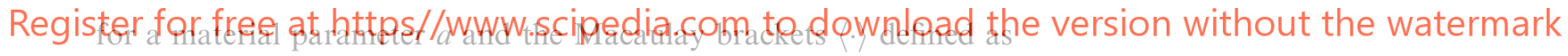

$$
\langle x\rangle:= \begin{cases}0 & \text { if } x \leqslant 0 \\ x & \text { if } x \geqslant 0\end{cases}
$$

The damage surface (2.8) is depicted in Fig. 1. The symbol $\boldsymbol{\sigma}^{\mathrm{d}}(=\boldsymbol{\sigma})$, with its corresponding deviatoric part $s^{\mathrm{d}}$ and hydrostatic pressure $p^{\mathrm{d}}$, refers to the stresses in the context of the damage model. We note that expression (2.8) is defined in terms of a homogeneous function of degree two on the stresses. As discussed in Remark 3.2.1 of Part I, this form assures a positive damage dissipation $\left(\tilde{\omega}_{0}^{\mathrm{d}}>0\right)$. The damage surface (2.8) can be understood as a linear combination of the distortional energy (proportional to $\left\|\boldsymbol{s}^{\mathrm{d}}\right\|^{2}$ ) and the

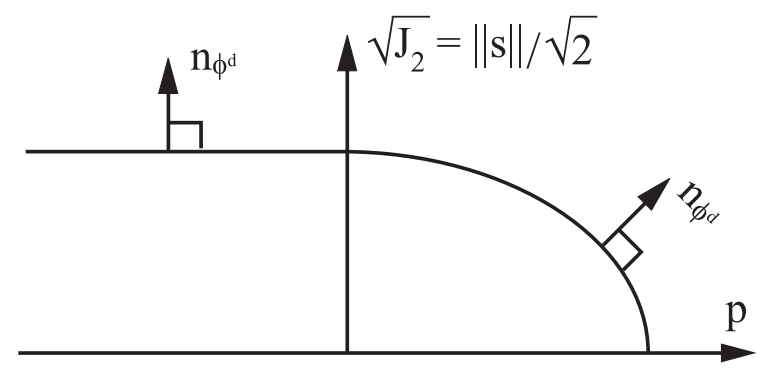

Fig. 1. A plastic damage model - pressure dependent damage surface. 
volumetric energy (proportional to $p^{\mathrm{d}^{2}}$ ). The latter is only considered for the case of volumetric tension $p^{d}>0$ through the use of the Macaulay (2.9). The motivation behind this choice is to model in this case a lack of evolution of the volumetric compliance when using the evolution equation

$$
\dot{\mathbf{D}}^{\mathrm{d}}=\gamma^{\mathrm{d}} \frac{\boldsymbol{n}_{\tilde{\phi}^{\mathrm{d}}} \otimes \boldsymbol{n}_{\tilde{\phi}^{\mathrm{d}}}}{\boldsymbol{n}_{\tilde{\phi}^{\mathrm{d}}}: \boldsymbol{\sigma}^{\mathrm{d}}} \quad \text { with } \boldsymbol{n}_{\tilde{\phi}^{\mathrm{d}}}:=\frac{\partial \tilde{\phi}^{\mathrm{d}}}{\partial \boldsymbol{\sigma}^{\mathrm{d}}},
$$

for the damage compliance $\mathbf{D}^{\mathrm{d}}$, as developed in Part I. This is the case $\operatorname{since} \operatorname{tr}\left(\boldsymbol{n}_{\phi^{\mathrm{d}}}\right.$ ) for $p^{\mathrm{d}} \leqslant 0$ (Fig. 1) with the evolution equation (2.10) still accounts for the damage degradation due to the distorsion of the voids. The inclusion of a cap surface in compression can be easily accommodated. The smoothness of the yield surface $(2.8)$ is to be noted.

The damage space $\mathscr{V}^{\mathrm{d}}$ is given in this case by the full space $\mathscr{S}$ of symmetric tensors, that is, the projection matrix $\mathbb{P}^{\mathrm{d}}$ onto the space of damage strains is simply $\mathbb{P}^{\mathrm{d}}=\mathbb{}$. The final evolution of the damage compliance is then obtained from Eq. (2.10) as

$$
\dot{\mathbf{D}}^{\mathrm{d}}=2 \gamma^{\mathrm{d}} \frac{\left(a s^{\mathrm{d}}+\frac{1}{3}\left\langle p^{\mathrm{d}}\right\rangle \mathbb{1}\right) \otimes\left(a s^{\mathrm{d}}+\frac{1}{3}\left\langle p^{\mathrm{d}}\right\rangle \mathbb{1}\right)}{a\left\|s^{\mathrm{d}}\right\|^{2}+\left\langle p^{\mathrm{d}}\right\rangle^{2}}
$$

\section{with the hardening/softening law}

$$
\dot{\alpha}^{\mathrm{d}}=\gamma^{\mathrm{d}} \text {. }
$$

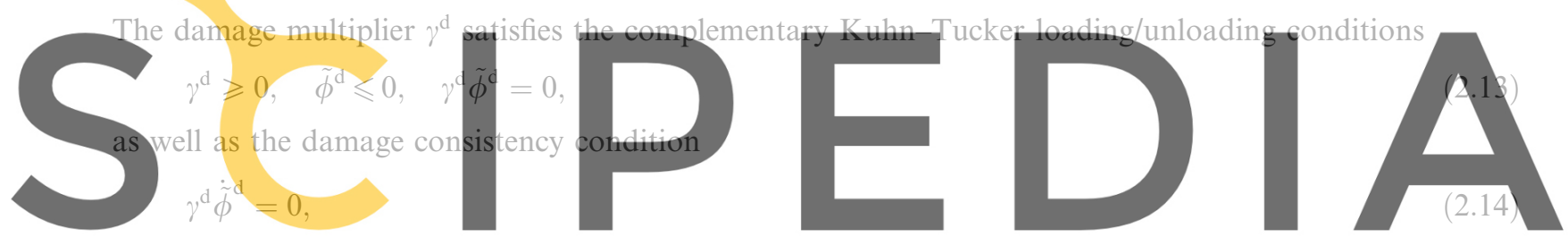

during persistent damage. The anisotropy of the damage compliance $\mathbf{D}^{\mathrm{d}}$ induced by a non-monotonic stress

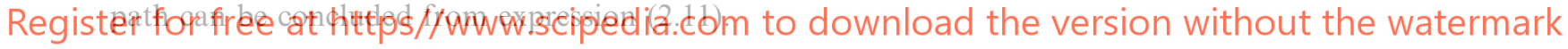

Remark 2.1. (1) For the particular case $a=0$, criteria (2.8) reduces to a purely volumetric damage model in terms of the hydrostatic pressure $p^{\mathrm{d}}$. In this case, the formalism introduced in Part I for the reduced characterization of the damage mechanism can be used with the damag space (2.8) defined by the single projection matrix $\left(n_{\mathrm{d}}=1\right)$

$$
\mathbb{P}^{\mathrm{d}}=\frac{1}{\sqrt{3}} \mathbf{1}
$$

The scaling in Eq. (2.15) is such that the orthonormality relation $\mathbb{P}^{\mathrm{d}}: \mathbb{P}^{\mathrm{d}}=1$ holds. Therefore, we have in this case

$$
\begin{aligned}
& \boldsymbol{s}^{\mathrm{d}}=\mathbb{P}^{\mathrm{d}}: \boldsymbol{\sigma}^{\mathrm{d}}=\frac{1}{\sqrt{3}} \operatorname{tr}\left(\boldsymbol{\sigma}^{\mathrm{d}}\right)=\sqrt{3} p^{\mathrm{d}}, \\
& \boldsymbol{\varepsilon}^{\mathrm{d}}=\mathbb{P}^{\mathrm{d}} e^{\mathrm{d}} \quad \text { for } e^{\mathrm{d}}=\frac{1}{\sqrt{3}} \operatorname{tr}\left(\boldsymbol{\varepsilon}^{\mathrm{d}}\right)=\frac{1}{\sqrt{3}} e_{\mathrm{v}}^{\mathrm{d}},
\end{aligned}
$$

for the pressure $p^{\mathrm{d}}$ and the damage volumetric strain $e_{\mathrm{v}}^{\mathrm{d}}:=\operatorname{tr}\left(\boldsymbol{\varepsilon}^{\mathrm{d}}\right)$. The damage evolution equation (2.11) reduces in this case to

$$
\dot{\mathbf{D}}^{\mathrm{d}}=\frac{1}{9} \dot{\mathrm{d}}_{\mathrm{v}}^{\mathrm{d}} \mathbf{1} \otimes \mathbf{1} \quad \text { with } \dot{\mathrm{d}}_{\mathrm{v}}^{\mathrm{d}}=2 \gamma^{\mathrm{d}}
$$


for the damage volumetric compliance $\mathrm{d}_{\mathrm{v}}^{\mathrm{d}}:=e_{\mathrm{v}}^{\mathrm{d}} / p^{\mathrm{d}}=3 \mathrm{~d}^{\mathrm{d}}$ with $\mathrm{d}^{\mathrm{d}}=e^{\mathrm{d}} / \mathbf{s}^{\mathrm{d}}$.

(2) Along the same lines, the hardening/softening law $\tilde{\omega}^{\mathrm{d}}\left(\alpha^{\mathrm{d}}\right)$ can be obtained experimentally by matching a particular test. Section 2.1.1 considers the common case of uniaxial tension test. As an alternative example, let $\hat{p}\left(e_{\mathrm{v}}^{\mathrm{d}}\right)$ be the pressure evolution obtained in a purely (tension) volumetric test of the porous metal, where

$$
e_{\mathrm{v}}^{\mathrm{d}}=e_{\mathrm{v}}-e_{\mathrm{v}}^{\mathrm{e}}\left(-e_{\mathrm{v}}^{\mathrm{p}}\right)
$$

for the applied volumetric strain $e_{\mathrm{v}}:=\operatorname{tr}[\varepsilon]$, measured elastic strain $e_{\mathrm{v}}^{\mathrm{e}}=p / \kappa^{\mathrm{e}}\left(\kappa^{\mathrm{e}}\right.$, the elastic bulk modulus), and measured (by unloading) plastic volumetric strain $e_{\mathrm{v}}^{\mathrm{p}}(=0$ in the assumed model for metals). The measure $e_{\mathrm{v}}^{\mathrm{d}}$ is related directly in Section 2.2 to the void fraction in the material. Integrating Eq. (2.18), we obtain

$$
\alpha^{\mathrm{d}}=\frac{1}{2} \mathrm{~d}_{\mathrm{v}}^{\mathrm{d}}=\frac{e_{\mathrm{v}}^{\mathrm{d}}}{2 \hat{p}\left(e_{\mathrm{v}}^{\mathrm{d}}\right)}
$$

after using $E q$. (2.12). The final hardening/softening law in Eq. (2.8) is then obtained as

$$
\tilde{\omega}^{\mathrm{d}}\left(\alpha^{\mathrm{d}}\right)=\hat{p}^{2}\left(\hat{e}_{\mathrm{v}}^{\mathrm{d}}\left(\alpha^{\mathrm{d}}\right)\right) \text {, }
$$

where the function $\hat{e}_{\mathrm{v}}^{\mathrm{d}}\left(\alpha^{\mathrm{d}}\right)$ is obtained by inverting the relation (2.20). In the numerical simulations presented in Section 4, the inversion of relation (2.20), giving $\alpha^{\mathrm{d}}$ in terms of the intermediate internal variable $e_{\mathrm{v}}^{\mathrm{d}}$ for a given hardening/softening law $\hat{p}\left(e_{\mathrm{v}}^{\mathrm{d}}\right)$, is done numerically through a Newton iterative scheme.

\section{1 . Model prediction in uniarial tension tests}
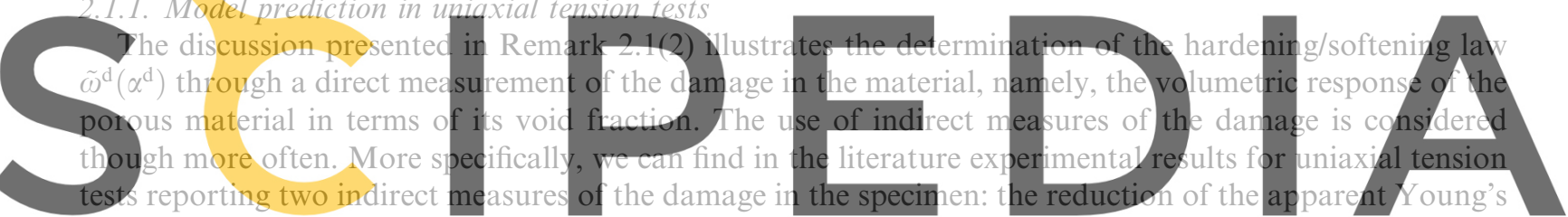

modulus (that is, the slope of the measured axial stress/strain relation upon unloading) and the apparent

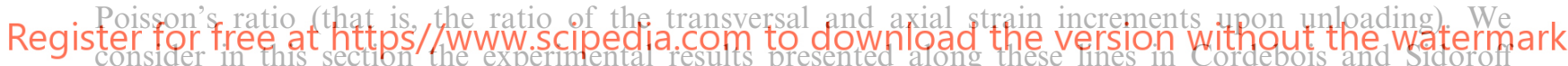

(1979), Lemaitre (1983) and Chow and Wang (1987) for a 2024-T3 aluminum alloy, as compiled by Hansen and Schreyer (1994), and compare them with the predictions of the material model under consideration. We refer to these references for a discussion of the test setup. Our goal in doing this is not to validate the damage surface (2.8) (the shape parameter $a$, in particular), since obviously additional tests are needed, but to illustrate different features of the damage effects incorporated in the material response by the proposed framework involving the non-standard decomposition of the strains in elastic, damage and plastic parts. Unfortunately, complete experimental investigations of the damage observed in metals after unloading in more general settings are lacking to a large extent (Hansen and Schreyer, 1994).

The particularization of the damage evolution equations (2.11) and (2.12) to the uniaxial tension stress state of interest (i.e., $\sigma_{11}=\sigma>0$ with all other components zero, $x_{1}$ being the loading direction) leads, upon straightforward algebraic manipulations and integration, to the relations

$$
\left\{\begin{array}{l}
D_{1111}^{\mathrm{d}}=\frac{2}{9}(1+6 a) \alpha^{\mathrm{d}}, \\
D_{1122}^{\mathrm{d}}=D_{2211}^{\mathrm{d}}=D_{1133}^{\mathrm{d}}=D_{3311}^{\mathrm{d}}=\frac{2}{9}(1-3 a) \alpha^{\mathrm{d}}, \\
D_{2222}^{\mathrm{d}}=D_{3333}^{\mathrm{d}}=D_{2233}^{\mathrm{d}}=D_{3322}^{\mathrm{d}}=\frac{2}{9} \frac{(1-3 a)^{2}}{(1+6 a)} \alpha^{\mathrm{d}} \\
\text { with all other components zero }
\end{array}\right.
$$

for the different components of the damage compliance in terms of the damage internal variable $\alpha^{\mathrm{d}}>0$. The axial damage compliance $D_{1111}^{\mathrm{d}}$ is denoted simply by $D^{\mathrm{d}} \equiv D_{1111}^{\mathrm{d}}$ in what follows. During persistent damage loading, the damage condition (2.8) holds and reads for the uniaxial state of interest 


$$
\sigma^{2}=\frac{9}{(1+6 a)} \tilde{\omega}^{\mathrm{d}}\left(\alpha^{\mathrm{d}}\right)=\frac{9}{1+6 a} \tilde{\omega}^{\mathrm{d}}\left(\frac{9 D^{\mathrm{d}}}{2(1+6 a)}\right) \Rightarrow D^{\mathrm{d}}=\hat{D}^{\mathrm{d}}(\sigma)
$$

giving the axial damage compliance $D^{\mathrm{d}}$ in terms of the axial stress $\sigma>0$. During unloading, the material model results on a constant damage compliance $D^{\mathrm{d}}$, fixed at its last value during loading. Denoting the axial damage strain by $\varepsilon^{\mathrm{d}} \equiv \epsilon_{11}^{\mathrm{d}}$, the general relation $\boldsymbol{\varepsilon}^{\mathrm{d}}=\mathbf{D}^{\mathrm{d}} \boldsymbol{\sigma}$ leads in this case to the axial relation

$$
\varepsilon^{\mathrm{d}}=\hat{\varepsilon}^{\mathrm{d}}(\sigma)=\hat{D}^{\mathrm{d}}(\sigma) \sigma
$$

in terms of the axial stress $\sigma$. Note that Eq. (2.24) holds both for loading and unloading.

Similarly, denoting the axial plastic strain by $\varepsilon^{\mathrm{p}} \equiv \varepsilon_{11}^{\mathrm{p}}$, the plastic evolution equations (2.3) and (2.4) and the yield condition (2.1) lead during plastic loading to the relations

$$
\left\{\begin{array}{l}
\varepsilon^{\mathrm{p}}=\alpha^{\mathrm{p}}, \\
|\sigma|=\tilde{y}^{\mathrm{p}}\left(\alpha^{\mathrm{p}}\right),
\end{array} \Rightarrow \varepsilon^{\mathrm{p}}=\hat{\varepsilon}^{\mathrm{p}}(\sigma),\right.
$$

$(\sigma>0)$, with $\varepsilon^{\mathrm{p}}$ fixed during unloading to its last value in loading. Combining these results, we obtain the relation

$$
\varepsilon=\hat{\varepsilon}^{\mathrm{e}}(\sigma)+\hat{\varepsilon}^{\mathrm{d}}(\sigma)+\hat{\varepsilon}^{\mathrm{p}}(\sigma)=E^{\mathrm{e}^{-1}} \sigma+\hat{D}^{\mathrm{d}}(\sigma) \sigma+\hat{\varepsilon}^{\mathrm{p}}(\sigma)
$$

for the total axial strain $\varepsilon \equiv \varepsilon_{11}$. Here, we denote by $E^{\mathrm{e}}$ the constant Young modulus characterizing the axial elastic response before damage appears $\left(E^{\mathrm{e}}=9 \kappa^{\mathrm{e}} \mu^{\mathrm{e}} /\left(3 \kappa^{\mathrm{e}}+\mu^{\mathrm{e}}\right)\right.$ in terms of the elastic moduli considered in
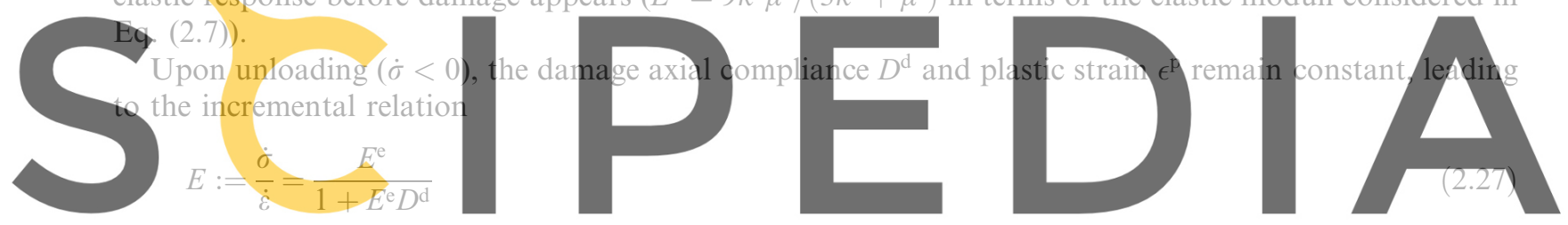

for the apparent Young's modulus E. Similarly, the Poisson ratio observed upon unloading is obtained Registeft fior fneejlatdmttps/quawwisoipedia.com to download the version without the watermark

$$
v:=-\frac{\dot{\epsilon}_{22}}{\dot{\epsilon}_{11}}=v^{\mathrm{e}} \frac{E}{E^{\mathrm{e}}}\left(1-\frac{E^{\mathrm{e}}}{v^{\mathrm{e}}} D_{2211}^{\mathrm{d}}\right)=v^{\mathrm{e}} \frac{1-\frac{E^{\mathrm{e}}}{v^{\mathrm{e}}} \frac{(1-3 a)}{(1+6 a)} D^{\mathrm{d}}}{1+E^{\mathrm{e}} D^{\mathrm{d}}}
$$

for the initial Poisson ratio $v^{\mathrm{e}}\left(v^{\mathrm{e}}=\left(3 \kappa^{\mathrm{e}}-2 \mu^{\mathrm{e}}\right) /\left(6 \kappa^{\mathrm{e}}+2 \mu^{\mathrm{e}}\right)\right.$ in terms of the elastic moduli considered in Eq. (2.7)).

These expressions allow one to relate the material laws $\tilde{y}^{\mathrm{p}}\left(\alpha^{\mathrm{p}}\right)$ and $\tilde{\omega}^{\mathrm{d}}\left(\alpha^{\mathrm{d}}\right)$ governing the evolution of the plastic and damage effects, respectively, with the axial stress/strain relation (2.26), and the Young modulus (2.27) and Poisson ratio (2.28) reduction observed in the response of the material due to damage. As an example, we consider the general power law

$$
\tilde{y}^{\mathrm{p}}\left(\alpha^{\mathrm{p}}\right)=y_{0}^{\mathrm{p}}\left[1+\frac{H^{\mathrm{p}}}{y_{0}^{\mathrm{p}}}\left(\alpha^{\mathrm{p}}\right)^{n_{\mathrm{p}}}\right]
$$

for the plastic part, in terms of the initial yield limit $y_{0}^{\mathrm{p}}$ (units of stress) and the hardening parameters $H^{\mathrm{p}}$ (units of stress) and $n_{\mathrm{p}}$ (non-dimensional). Note that the equivalent plastic strain $\alpha^{\mathrm{p}}$ is non-dimensional. Similarly, we consider the power law

$$
\tilde{\omega}^{\mathrm{d}}\left(\alpha^{\mathrm{d}}\right)=\tilde{\omega}_{0}^{\mathrm{d}}\left[1+\left(H^{\mathrm{d}} \alpha^{\mathrm{d}}\right)^{n_{\mathrm{d}}}\right]
$$

for the damage part, in terms of the damage hardening parameter $H^{\mathrm{d}}$ (units of stress) and $n_{\mathrm{d}}$ (non-dimensional). The material parameter $\tilde{\omega}_{0}^{d}$ measuring the threshold of damage is re-scaled as $\tilde{\omega}_{0}^{\mathrm{d}}=\sigma_{0}^{\mathrm{d}^{2}}(1+6 a) / 9$ 
for the threshold damage stress $\sigma_{0}^{\mathrm{d}}$ (units of stress). Note that the damage hardening internal variable $\alpha^{\mathrm{d}}$ has units of compliance (stress inverse) in the model under consideration.

Figs. 2 and 3 show the results obtained when considering the power laws (2.29) and (2.30) with the material parameters of Table 1 . The experimental results reported in the aforementioned references for a 2024-T3 aluminum alloy are included. The chosen parameters have been determined by simple trial and error. A good qualitative matching can be observed. In particular, we note not only the reduction of the

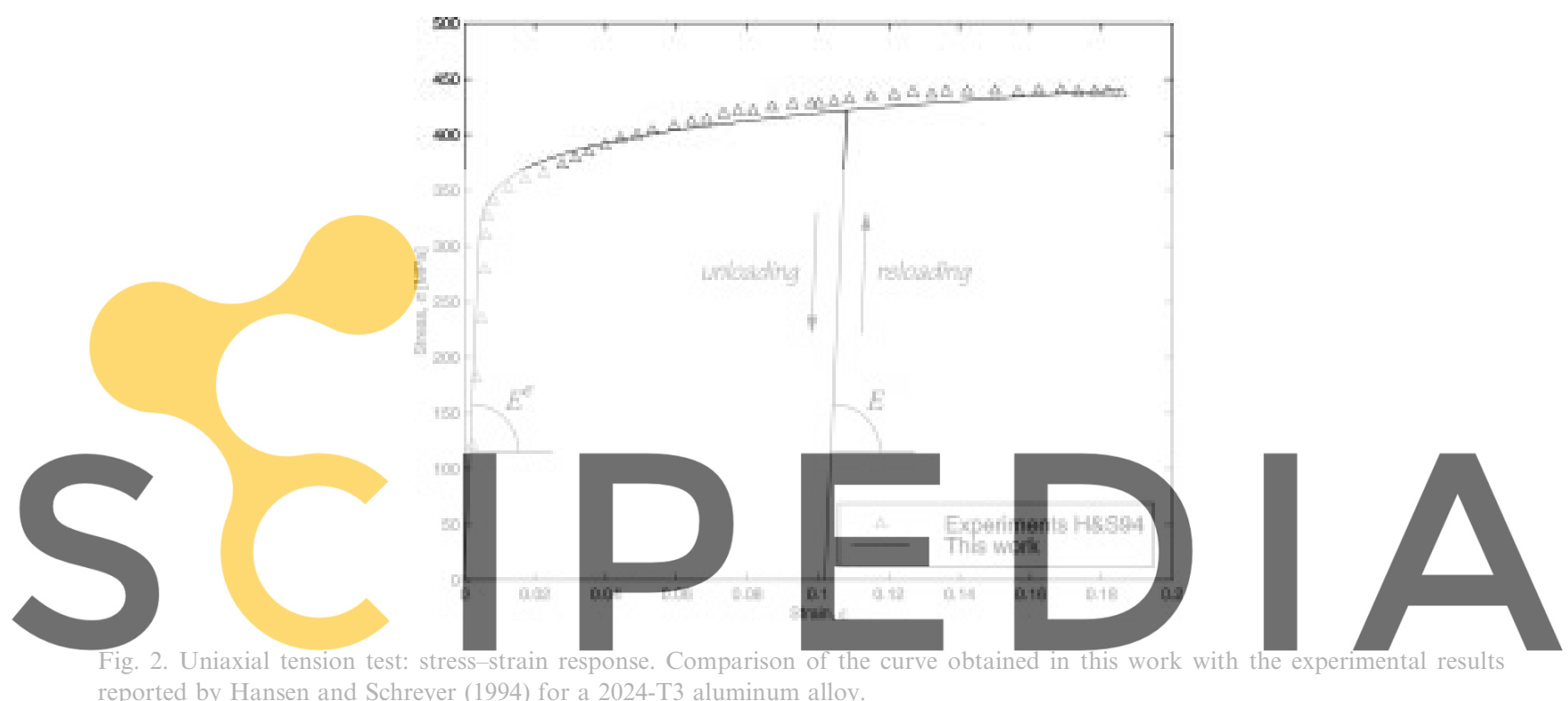

Register for free at https//www.scipedia.com to download the version without the watermark
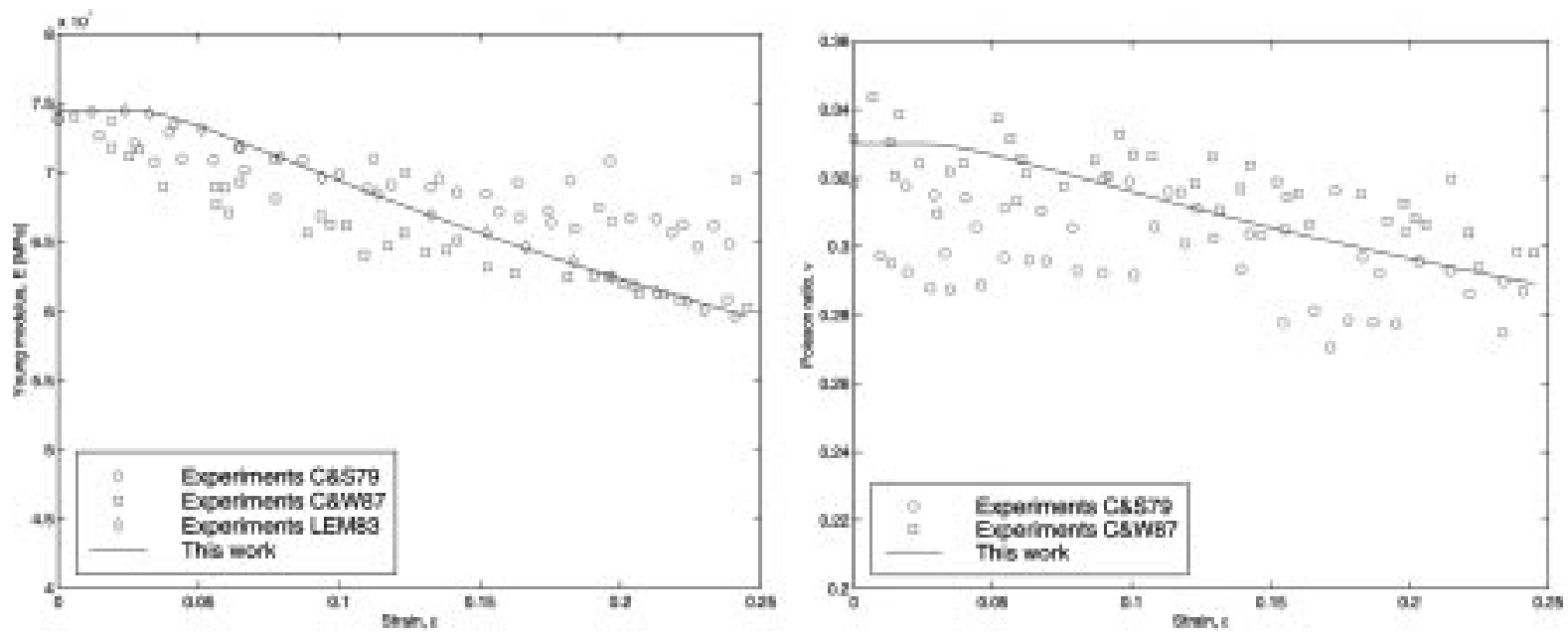

Fig. 3. Uniaxial tension test: evolutions of the apparent Young modulus $E$ and Poisson ratio $v$. Comparison of the response predicted with the proposed model and experimental results reported for a 2024-T3 aluminum alloy: "C\&S79" - Cordebois and Sidoroff (1979), "C\&W87" - Chow and Wang (1987) and "LEM83" - Lemaitre (1983) (experimental data provided by Hansen and Schreyer (1994)). 
Table 1

Uniaxial tension tests on a 2024-T3 aluminum alloy - material parameters

\begin{tabular}{lll}
\hline Initial Young's modulus & $E^{\mathrm{e}}$ & $74,500 \mathrm{MPa}$ \\
Initial Poisson's ratio & $v^{\mathrm{e}}$ & 0.33 \\
Initial yield limit & $y_{0}^{\mathrm{p}}$ & $270 \mathrm{MPa}$ \\
Plastic hardening exponent & $n_{\mathrm{p}}$ & 0.2 \\
Plastic hardening parameter & $H^{\mathrm{p}}$ & $240 \mathrm{MPa}$ \\
Initial damage threshold & $\sigma_{0}^{\mathrm{d}}$ & $380 \mathrm{MPa}$ \\
Damage exponent & $n_{\mathrm{d}}$ & 0.5 \\
Damage hardening parameter & $H^{\mathrm{d}}$ & $43,163 \mathrm{MPa}$ \\
Damage surface parameter & $a$ & 0.5 \\
\hline
\end{tabular}

Young modulus with the strain due to the presence of damage in the material, but also the reduction of the apparent Poisson ratio. This last situation reflects the anisotropy induced by the proposed damage model in response of the material. A simple isotropic damage model based on a scalar damage variable, as considered in Section 3.2.2 of Part I of this work, cannot predict this reduction of the Poisson ratio (Hansen and Schreyer, 1994).

Additional features of the model considered herein and, in particular, of the damage surface (2.8) in general non-monotonic uniaxial stress states are studied in Section 4 through a series of numerical simulations. These analyses consider also the characterization of damage deactivation (or unilateral damage) presented in the following section as it occurs after load reversing, a situation not considered in the aforementioned experimental resul

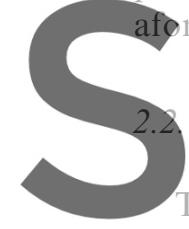

Unilateral damage

The damage surface (2.8) defines the evolution of the damag
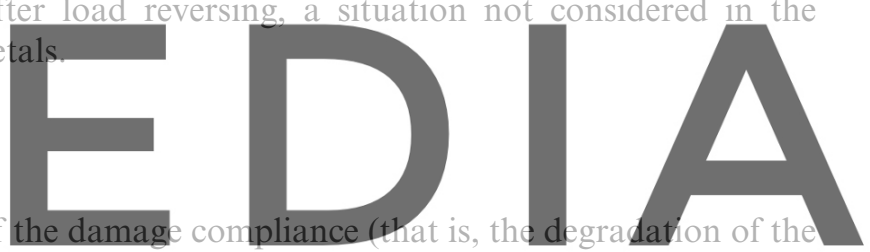

material's stiffness), as defined in Eq. (2.11) for the assumed associated case through its normal $n_{\hat{\phi}^{d}}$. The

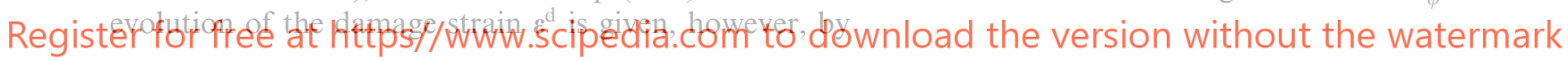

$$
\dot{\varepsilon}^{\mathrm{d}}=\left(\mathrm{D}^{\mathrm{d}} \sigma^{\mathrm{d}}\right)=\gamma^{\mathrm{d}} n_{\phi^{\mathrm{d}}}+\mathrm{D}^{\mathrm{d}} \dot{\sigma}^{\mathrm{d}}
$$

after using Eq. (2.10) for the rate of the damage compliance. The last term in Eq. (2.31) reflects the recoverable character of the damage strain for the case $\gamma^{\mathrm{d}}=0$ of no further damage. This independent nature of the evolution of the damage strain and of the damage compliance allows the introduction of additional constraints on the damage strain.

In particular, the closing of voids and subsequent recovery of stiffness in the material (an effect generally referred to as unilateral damage) can be easily modeled by imposing the constraint

$$
e_{\mathrm{v}}^{\mathrm{d}}:=\operatorname{tr}\left[\boldsymbol{\varepsilon}^{\mathrm{d}}\right] \geqslant 0
$$

for the volumetric part of the damage strain. Since $\operatorname{tr}\left[\varepsilon^{\mathrm{p}}\right]=0$ for the assumed plastic model, the kinematic decomposition (2.19) reads

$$
e_{\mathrm{v}}=e_{\mathrm{v}}^{\mathrm{e}}+e_{\mathrm{v}}^{\mathrm{d}}=\underbrace{\frac{\Delta v^{\mathrm{e}}}{v_{0}}}_{e_{\mathrm{v}}^{\mathrm{e}}}+\underbrace{\frac{\Delta v^{\mathrm{d}}}{v_{0}}}_{e_{\mathrm{v}}^{\mathrm{d}}}
$$

for an elastic increment $\Delta v^{\mathrm{e}}$ of an initial volume $v_{0}$ and the volume of voids $v^{\mathrm{d}} \equiv \Delta v^{\mathrm{d}}$ (assuming $v^{\mathrm{d}} \equiv 0$ initially). In this notation, the volume fraction of voids $f:=v^{\mathrm{d}} / v_{0}$ is obtained as 


$$
f=\frac{e_{\mathrm{v}}^{\mathrm{e}}+e_{\mathrm{v}}^{\mathrm{d}}}{1+e_{\mathrm{v}}^{\mathrm{e}}+e_{\mathrm{v}}^{\mathrm{d}}}
$$

Thus, after full unloading $p^{\mathrm{d}}=0$ so $e_{\mathrm{v}}^{\mathrm{e}}=0$ (reference state corresponding to an unstressed state) and hence

$$
f_{p=0}=\frac{e_{\mathrm{v}}^{\mathrm{d}}}{1+e_{\mathrm{v}}^{\mathrm{d}}} .
$$

Constraint (2.32) then imposes a non-negative void fraction $f_{p=0} \geqslant 0$, as is physically expected.

The unilateral constraint (2.32) is easily accommodated in the previous developments through the method of Lagrange multipliers (Luenberger, 1984). In this context, following the same arguments as in Section 2.2 of Part I for the derivation of the general damage framework, the added unilateral constraint (2.32) leads to the modified stress-strain damage relation

$$
\left\{\begin{array}{l}
\boldsymbol{\sigma}^{\mathrm{d}}=\frac{\partial \widehat{W}^{\mathrm{d}}}{\partial \boldsymbol{\varepsilon}^{\mathrm{d}}}-\lambda^{\mathrm{d}} \mathbf{1}, \\
\lambda^{\mathrm{d}} \geqslant 0, \quad \operatorname{tr}\left[\boldsymbol{\varepsilon}^{\mathrm{d}}\right] \geqslant 0, \quad \lambda^{\mathrm{d}} \operatorname{tr}\left[\boldsymbol{\varepsilon}^{\mathrm{d}}\right]=0
\end{array}\right.
$$

for the Lagrange multiplier $\lambda^{\mathrm{d}}$. For the quadratic damage potential of interest, the first part of relation (2.36) reduces to

$$
\boldsymbol{\sigma}^{\mathrm{d}}=\widehat{\boldsymbol{\sigma}}^{\mathrm{d}}-\lambda^{\mathrm{d}} \mathbf{1} \text { for } \widehat{\boldsymbol{\sigma}}^{\mathrm{d}}:=\mathbf{D}^{\mathrm{d}^{-1}} \boldsymbol{\varepsilon}^{\mathrm{d}} .
$$

After imposing constraint (2.32), relations (2.36) then lead to the closed-form expression

$$
\lambda^{\mathrm{d}}=\frac{\left\langle-\mathbf{1}: \mathbf{D}^{\mathrm{d}} \boldsymbol{\sigma}^{\mathrm{d}}\right\rangle}{\mathbf{1}: \mathbf{D}^{\mathrm{d}} \mathbf{1}}
$$

for the Macaulay brackets $\langle\cdot\rangle$ defined in Eq. (2.9). Combining Eq. (2.36) with Eq. (2.38), we obtain after a straightforward calculation

$$
\boldsymbol{\varepsilon}^{\mathrm{d}}=\widehat{\mathbf{D}}^{\mathrm{d}} \boldsymbol{\sigma}^{\mathrm{d}} \text { for } \widehat{\mathbf{D}}^{\mathrm{d}}=\mathbf{D}^{\mathrm{d}}-\frac{\mathbf{D}^{\mathrm{d}} \mathbf{1} \otimes \mathbf{D}^{\mathrm{d}} \mathbf{1}}{\mathbf{1}: \mathbf{D}^{\mathrm{d}} \mathbf{1}} H\left(-\mathbf{1}: \mathbf{D}^{\mathrm{d}} \boldsymbol{\sigma}^{\mathrm{d}}\right),
$$

where $H(\cdot)$ denotes the Heaviside jump function $(H(x)=0$ for $x<0$ and $H(x)=1$ for $x \geqslant 0)$. We note the singularity of $\widehat{\mathbf{D}}^{\mathrm{d}}$ on volumetric fields (i.e., $\widehat{\mathbf{D}}^{\mathrm{d}} \mathbf{1}=0$ ) upon void closing, reflecting the full recovery of the volumetric stiffness in the material after this happens. Since for the assumed von Mises model of plasticity the volumetric response remains elastic, the elastic stiffness is recovered in the volumetric response. For the case of the isotropic elastic response (Eq. (2.7)), we have

$$
e_{\mathrm{v}}=e_{\mathrm{v}}^{\mathrm{e}}+e_{\mathrm{v}}^{\mathrm{d}}=\kappa^{\mathrm{e}^{-1}} p+\boldsymbol{\sigma}^{\mathrm{d}}: \widehat{\mathbf{D}}^{\mathrm{d}} \mathbf{1}
$$

recovering the elastic response $p=\kappa^{\mathrm{e}} e_{\mathrm{v}}$ upon void closing.

If the damage evolution equations (2.10) are maintained with a damage surface (2.8) in terms of the stresses $\sigma^{\mathrm{d}}$, the resulting damage rate relation reads

$$
\dot{\boldsymbol{\varepsilon}}^{\mathrm{d}}=\widehat{\mathbf{D}}_{\mathrm{lu}}^{\mathrm{d}} \dot{\boldsymbol{\sigma}}^{\mathrm{d}} \quad \text { with } \widehat{\mathbf{D}}_{\mathrm{lu}}^{\mathrm{d}}= \begin{cases}\widehat{\mathbf{D}}^{\mathrm{d}} & \text { for damage unloading, } \\ \widehat{\mathbf{D}}^{\mathrm{d}}+\frac{1}{\Delta^{\mathrm{d}}} \widehat{\boldsymbol{n}}_{\tilde{\phi}^{\mathrm{d}}} \otimes \boldsymbol{n}_{\tilde{\phi}^{\mathrm{d}}} & \text { for damage loading, }\end{cases}
$$

where

$$
\Delta^{\mathrm{d}}=\boldsymbol{n}_{\tilde{\phi}^{\mathrm{d}}}: \mathbf{C}^{\mathrm{d}} \boldsymbol{n}_{\tilde{\phi}^{\mathrm{d}}}+K^{\mathrm{d}}
$$

for the damage stiffness $\mathbf{C}^{\mathrm{d}}=\mathbf{D}^{\mathrm{d}^{-1}}$, the hardening/softening tangent modulus $K^{\mathrm{d}}:=\partial \tilde{\omega}^{\mathrm{d}} / \partial \alpha^{\mathrm{d}}$, and 


$$
\widehat{\boldsymbol{n}}_{\tilde{\phi}^{\mathrm{d}}}=\left(1+\lambda^{\mathrm{d}} \frac{\boldsymbol{n}_{\tilde{\phi}^{\mathrm{d}}}: \mathbf{1}}{\boldsymbol{n}_{\tilde{\phi}^{\mathrm{d}}}: \boldsymbol{\sigma}^{\mathrm{d}}}\right) \boldsymbol{n}_{\tilde{\phi}^{\mathrm{d}}}+\lambda^{\mathrm{d}} \frac{\boldsymbol{n}_{\tilde{\phi}^{\mathrm{d}}}: \mathbf{1}}{\boldsymbol{n}_{\tilde{\phi}^{\mathrm{d}}}: \boldsymbol{\sigma}^{\mathrm{d}}}\left(\frac{\left(\boldsymbol{n}_{\tilde{\phi}^{\mathrm{d}}}^{\mathrm{d}}: \boldsymbol{\sigma}^{\mathrm{d}}\right)^{2}}{\mathbf{1}: \mathbf{D}^{\mathrm{d}} \boldsymbol{\sigma}^{\mathrm{d}}}-\frac{\boldsymbol{n}_{\tilde{\phi}^{\mathrm{d}}}: \mathbf{1}}{\mathbf{1}: \mathbf{D}^{\mathrm{d}} \mathbf{1}}\right) \mathbf{D}^{\mathrm{d}} \mathbf{1} .
$$

We note that, in general, $\boldsymbol{n}_{\tilde{\phi}^{\mathrm{d}}} \neq \widehat{\boldsymbol{n}}_{\tilde{\phi}^{\mathrm{d}}}$, leading then to an unsymmetric tangent relation when damage loading occurs. After Eq. (2.43), we can observe that symmetry holds if $\boldsymbol{n}_{\tilde{\phi}^{\mathrm{d}}}: \mathbf{1}=0$. For the damage surface (2.8), this is the case for negative pressure $p \leqslant 0$. We note, however, that in general the closing of the voids is given by constraint (2.32) on the strains and is not controlled directly by the stresses. Therefore, an unsymmetric tangent appears when closing occurs while $p>0$. We also note that this situation does not occur for the particular case $a=0$, with the corresponding reduced volumetric damage mechanism described in Remark 2.1. In this case, Eq. (2.18) identifies the scalar relation $e_{\mathrm{v}}^{\mathrm{d}}=\mathrm{d}_{\mathrm{v}}^{\mathrm{d}} p$, so the closing of voids only occurs for $p \leqslant 0$.

Remark 2.2. (1) A formulation with symmetric tangent can be recovered as follows. When constraint (2.32) is taken in account, the complimentary energy reads

$$
\chi^{\mathrm{d}}\left(\boldsymbol{\sigma}^{\mathrm{d}} ; \mathbf{D}^{\mathrm{d}}, \alpha^{\mathrm{d}}\right):=\max _{\substack{\varepsilon^{\mathrm{d}} \\ \operatorname{tr}\left[\boldsymbol{\varepsilon}^{\mathrm{d}}\right] \geqslant 0}}\left\{\boldsymbol{\sigma}^{\mathrm{d}}: \boldsymbol{\varepsilon}^{\mathrm{d}}-\widehat{W}^{\mathrm{d}}\left(\mathbf{e}^{\mathrm{d}} ; \mathscr{I}^{\mathrm{d}}\right)\right\}=\frac{1}{2} \widehat{\boldsymbol{\sigma}}^{\mathrm{d}}: \mathbf{D}^{\mathrm{d}} \widehat{\boldsymbol{\sigma}}^{\mathrm{d}}-\mathscr{H}^{\mathrm{d}}\left(\alpha^{\mathrm{d}}\right),
$$

after noting the equality $\boldsymbol{\sigma}^{\mathrm{d}}: \mathbf{D}^{\mathrm{d}} \widehat{\boldsymbol{\sigma}}^{\mathrm{d}}=\widehat{\boldsymbol{\sigma}}^{\mathrm{d}}: \mathbf{D}^{\mathrm{d}} \widehat{\boldsymbol{\sigma}}^{\mathrm{d}}$ obtained after some algebraic manipulations using relations (2.36). The stresses $\widehat{\boldsymbol{\sigma}}^{\mathrm{d}}$ have been defined in Eq. (2.37). Therefore, the damage dissipation (Section 2.2 of Part I) is given by

$$
\mathscr{D}^{\mathrm{d}}=\frac{1}{2} \widehat{\boldsymbol{\sigma}}^{\mathrm{d}}: \dot{\mathbf{D}}^{\mathrm{d}} \widehat{\boldsymbol{\sigma}}^{\mathrm{d}}+q^{\mathrm{d}} \dot{\alpha}^{\mathrm{d}}
$$

in terms of the stress $\widehat{\boldsymbol{\sigma}}^{\mathrm{d}}$. After considering a damage surface of the form

$$
\widehat{\phi}^{\mathrm{d}}\left(\widehat{\boldsymbol{\sigma}}^{\mathrm{d}}, q^{\mathrm{d}}\right) \leqslant 0
$$

(that is, replacing $\boldsymbol{\sigma}^{\mathrm{d}}$ by $\widehat{\boldsymbol{\sigma}}^{\mathrm{d}}$ in Eq. (2.8) for the case of interest) and the corresponding damage evolution equation

$$
\dot{\mathbf{D}}^{\mathrm{d}}=\gamma^{\mathrm{d}} \frac{\boldsymbol{n}_{\hat{\phi}^{\mathrm{d}}} \otimes \boldsymbol{n}_{\hat{\phi}^{\mathrm{d}}}}{\boldsymbol{n}_{\hat{\phi}^{\mathrm{d}}}: \widehat{\boldsymbol{\sigma}}^{\mathrm{d}}} \quad \text { for } \boldsymbol{n}_{\hat{\phi}^{\mathrm{d}}}:=\frac{\partial \widehat{\phi}^{\mathrm{d}}}{\partial \widehat{\boldsymbol{\sigma}}^{\mathrm{d}}}
$$

(note that $\widehat{\boldsymbol{n}}_{\tilde{\phi}^{\mathrm{d}}} \neq \boldsymbol{n}_{\hat{\phi}^{\mathrm{d}}}$ ), we recover after an involved calculation the symmetric tangent relation

$$
\dot{\boldsymbol{\varepsilon}}^{\mathrm{d}}=\widehat{\mathbf{D}}_{\mathrm{lu}}^{\mathrm{d}} \dot{\boldsymbol{\sigma}}^{\mathrm{d}} \quad \text { with } \widehat{\mathbf{D}}_{\mathrm{lu}}^{\mathrm{d}}=\mathbf{D}_{\mathrm{lu}}^{\mathrm{d}}-\frac{\mathbf{D}_{\mathrm{lu}}^{\mathrm{d}} \mathbf{1} \otimes \mathbf{D}_{\mathrm{lu}}^{\mathrm{d}} \mathbf{1}}{\mathbf{1}: \mathbf{D}_{\mathrm{lu}}^{\mathrm{d}} \mathbf{1}}
$$

for the original tangent compliance $\mathbf{D}_{\mathrm{lu}}^{\mathrm{d}}=\mathbf{C}_{\mathrm{lu}}^{\mathrm{d}^{-1}}$, with $\mathbf{C}_{\mathrm{lu}}$ given by

$$
\mathbf{C}_{\mathrm{lu}}^{\mathrm{d}}= \begin{cases}\mathbf{C}^{\mathrm{d}} & \text { for (damaged) unloading, } \\ \mathbf{C}^{\mathrm{d}}-\frac{1}{\Delta^{\mathrm{d}}} \mathbf{C}^{\mathrm{d}} \boldsymbol{n}_{\tilde{\phi}^{\mathrm{d}}} \otimes \mathbf{C}^{\mathrm{d}} \boldsymbol{n}_{\tilde{\phi}^{\mathrm{d}}}, & \text { for (persistent damage) loading }\end{cases}
$$

with $\Delta^{\mathrm{d}}$ defined in Eq. (2.42).

(2) A penalty regularization of the unilaterally constrained relations (2.36) is obtained as

$$
\boldsymbol{\sigma}^{\mathrm{d}}=\frac{\partial \widehat{W}^{\mathrm{d}}}{\partial \boldsymbol{\varepsilon}^{\mathrm{d}}}-\lambda^{\mathrm{d}} \mathbf{1} \quad \text { with } \lambda^{\mathrm{d}}=\left\langle-\rho_{\mathrm{p}} \operatorname{tr}\left[\boldsymbol{\varepsilon}^{\mathrm{d}}\right]\right\rangle
$$

for a scalar penalty parameter $\rho_{\mathrm{p}}>0$. In this case, the damage compliance (2.47) reads 


$$
\widehat{\mathbf{D}}^{\mathrm{d}}=\mathbf{D}^{\mathrm{d}}-\frac{\rho_{\mathrm{p}} H\left(e_{\mathrm{v}}^{\mathrm{d}}\right)}{1+\rho_{\mathrm{p}} \mathbf{1}: \mathbf{D}^{\mathrm{d}} \mathbf{1}} \mathbf{D}^{\mathrm{d}} \mathbf{1} \otimes \mathbf{D}^{\mathrm{d}} \mathbf{1}
$$

with a secant stiffness given by its inverse

$$
\widehat{\mathbf{C}}^{\mathrm{d}}=\widehat{\mathbf{D}}^{\mathrm{d}^{-1}}=\mathbf{C}^{\mathrm{d}}-\rho_{\mathrm{p}} H\left(e_{\mathrm{v}}^{\mathrm{d}}\right) \mathbf{1} \otimes \mathbf{1} .
$$

For the isotropic elastic case considered in Eq. (2.40), we have

$$
e_{\mathrm{v}}=\kappa^{\mathrm{e}^{-1}} p+\frac{1}{1+H\left(e_{\mathrm{v}}^{\mathrm{d}}\right) \rho_{\mathrm{p}} \mathbf{1}: \mathbf{D}^{\mathrm{d}} \mathbf{1}} \mathbf{1}: \mathbf{D}^{\mathrm{d}} \boldsymbol{\sigma}^{\mathrm{d}}
$$

after a simple calculation. Physically, not all the elastic stiffness is recovered upon closing in this regularized case.

(3) We note that all the formulations of damage deactivation presented above, namely, the original unsymmetric formulation presented in this section or the symmetrized and regularized formulations of Remarks 2.2(1) and (2), respectively, lead to a continuous stress response. This situation is to be contrasted with deactivation laws based on the stress or total strain (Hansen and Schreyer, 1995). This situation is to be traced again to the direct use of the damage strains $\boldsymbol{\varepsilon}^{\mathrm{d}}$ and the enforcement of the physically motivated constraints of damage deactivation on them. These ideas are illustrated in Section 4 with several numerical simulations.

\section{Numerical integration}

In this section, we develop the numerical integration of the damage models developed in the previous sections. In particular, Section 3.1 considers the general framework developed in Section 1 of Part I, considering the existence of multiple damage mechanisms. The presentation of these developments in this more general framework allows us to show more clearly the main advantage of the proposed numerical formulation maintaining the damage strains, namely, the modularity in the consideration of different deformation mechanisms, including plasticity and damage. In this context, Section 3.2 summarizes the return mapping algorithm considered in the integration of the elastoplastic model. The numerical integration of a general damage mechanism, with a particular application to the damage model considered in Section 2, is developed in Section 3.3. Representative numerical simulations illustrating the performance of the proposed numerical formulation are included in Section 4.

\subsection{General formulation}

The numerical integration of the general plastic damage is presented herein for a typical time (load) increment $\left[t_{n}, t_{n+1}\right]\left(\Delta t=t_{n+1}-t_{n}\right)$ in a standard Newton-Raphson type scheme for solving the resulting non-linear boundary value problem. In the context of the finite element method, the forthcoming relations occur at each quadrature point for given strains $\boldsymbol{\varepsilon}_{n}$ and $\boldsymbol{\varepsilon}_{n+1}$ at $t_{n}$ and $t_{n+1}$, respectively, the latter corresponding to the current iteration of the aforementioned Newton-Raphson procedure. The strain-driven structure of the final numerical scheme, as needed for the efficient implementation of displacement-type finite element methods, is to be noted in this respect. The goal of the integration algorithm is then to find the corresponding stresses $\sigma_{n+1}$ and the update of the internal plastic $\mathscr{I}_{n+1}^{\mathrm{p}}$ and damage $\mathscr{I}_{n+1}^{d_{\mathrm{I}}}\left(d_{\mathrm{I}}=1, n_{\text {dam }}\right)$ variables from its (given) values $\mathscr{I}_{n}^{\mathrm{p}}$ and $\mathscr{I}_{n}^{d_{\mathrm{I}}}$ at $t_{n}$. For convenience, we assume that the (reduced) damage strains $\boldsymbol{e}_{n}^{d_{\mathrm{I}}}$ for each damage mechanism $d_{\mathrm{I}}=1, n_{\mathrm{dam}}$ are also stored in the database, although their values are not strictly required. Here we use the same notation as in Part I. In particular, the damage strain $\boldsymbol{\varepsilon}^{\mathrm{d}}$ is decomposed as 


$$
\boldsymbol{\varepsilon}^{\mathrm{d}}=\sum_{d_{\mathrm{I}}=1}^{n_{\mathrm{dam}}} \boldsymbol{\varepsilon}^{d_{\mathrm{I}}} \quad \text { for } \boldsymbol{\varepsilon}^{d_{\mathrm{I}}}=\mathbb{P}^{d_{\mathrm{I}}^{\mathrm{T}}} \boldsymbol{e}^{d_{\mathrm{I}}} \in \mathbb{R}^{n_{d_{\mathrm{I}}}}
$$

in terms of the projection matrices defining the reduced damage space $\mathscr{V}^{d_{\mathrm{I}}}$, with the stresses associated with the damage mechanism defined by

$$
\boldsymbol{\sigma}^{d_{\mathrm{I}}}=\mathbb{P}^{d_{\mathrm{I}}^{\mathrm{T}}} \boldsymbol{s}^{d_{\mathrm{I}}} \quad \text { for } \boldsymbol{s}^{d_{\mathrm{I}}}=\mathbb{P}^{d_{\mathrm{I}}}: \boldsymbol{\sigma} \in \mathbb{R}^{n_{d_{\mathrm{I}}}}
$$

for each damage mechanism $d_{\mathrm{I}}=1, n_{\mathrm{dam}}\left(\boldsymbol{s}^{\mathrm{d}} \equiv \boldsymbol{\sigma}^{\mathrm{d}}, \mathbf{e}^{\mathrm{d}} \equiv \boldsymbol{\varepsilon}^{\mathrm{d}}\right.$ and $n_{\mathrm{dam}}=1$ in the particular damage model developed in Section 2).

The algorithm developed here is based on $n_{\mathrm{d}}^{\text {total }}=\sum_{d_{\mathrm{I}}=1}^{n_{\mathrm{dam}}} n_{d_{\mathrm{I}}}$, equilibrium relations pertaining to the second part of Eq. (3.2), written in residual form as

$$
\boldsymbol{R}^{d_{\mathrm{I}}}\left(\boldsymbol{e}_{n+1}^{d_{\mathrm{I}}}\right):=\mathbb{P}^{d_{\mathrm{I}}}: \boldsymbol{\sigma}_{n+1}^{\mathrm{ep}}-\boldsymbol{s}_{n+1}^{d_{\mathrm{I}}}
$$

between the stresses

$$
\boldsymbol{\sigma}_{n+1}^{\mathrm{ep}}=\widehat{\boldsymbol{\sigma}}^{\mathrm{ep}} \underbrace{\left(\boldsymbol{\varepsilon}_{n+1}-\boldsymbol{\varepsilon}_{n+1}^{\mathrm{p}}-\sum_{d_{\mathrm{J}}=1}^{n_{\mathrm{dam}}} \mathbb{P}^{d_{J}^{\mathrm{T}}} \mathbf{e}_{n+1}^{d_{\mathrm{J}}} ; \mathscr{I}_{n+1}^{\mathrm{p}}\right)}_{\varepsilon_{n+1}^{\mathrm{e}}}\left(=\frac{\partial W^{\mathrm{e}}}{\partial \boldsymbol{\varepsilon}^{\mathrm{e}}}\right)
$$

given by the elastoplastic in terms of the elastic potential $W^{\mathrm{e}}(\cdot)$ model depending on the updated plastic strain $\boldsymbol{\varepsilon}_{n+1}^{\mathrm{p}}$ and other plastic internal variables $\mathscr{I}_{n+1}^{\mathrm{p}}$ and the (reduced) damage stresses

$$
\boldsymbol{s}_{n+1}^{d_{\mathrm{I}}}=\widehat{\boldsymbol{s}^{d_{\mathrm{I}}}}\left(\boldsymbol{e}_{n+1}^{d_{\mathrm{I}}} ; \mathscr{I}_{n+1}^{d_{\mathrm{I}}}\right) \quad\left(=\frac{\partial \widehat{W}^{d_{\mathrm{I}}}}{\partial \boldsymbol{e}^{d_{\mathrm{I}}}}\right)
$$

for the updated damage internal variables $\mathscr{I}_{n+1}^{d_{\mathrm{I}}}$ associated to each damage mechanism. We point out that the final residuals (3.3) are considered to be explicit functions of the $n_{\mathrm{d}}^{\text {total }}$ damage strain parameters $\boldsymbol{e}_{n+1}^{d_{\mathrm{I}}}$ $\left(d_{\mathrm{I}}=1, n_{\mathrm{dam}}\right)$.

To solve the residual equations $\boldsymbol{R}^{d_{1}}=0$, we set the Newton scheme

$$
\boldsymbol{R}^{d_{\mathrm{I}}}\left(\boldsymbol{e}_{n+1, k}^{\mathrm{d}}\right)+\left.\sum_{d_{\mathrm{J}}=1}^{n_{\mathrm{dam}}} \frac{\partial \boldsymbol{R}^{d_{\mathrm{I}}}}{\partial \boldsymbol{e}^{d_{\mathrm{I}}}}\right|_{n+1, k} \Delta \boldsymbol{e}_{n+1, k}^{d_{\mathrm{J}}}=0 \quad \text { for } d_{\mathrm{I}}=1, n_{\mathrm{dam}}
$$

for an iteration index $k$ and with the incremental relation

$$
\boldsymbol{e}_{n+1, k+1}^{d_{\mathrm{I}}}=\boldsymbol{e}_{n+1, k}^{d_{\mathrm{I}}}+\Delta \boldsymbol{e}_{n+1, k}^{d_{\mathrm{I}}} \quad \text { for } d_{\mathrm{I}}=1, n_{\mathrm{dam}}
$$

initialized, for example, by $\boldsymbol{e}_{n+1,0}^{d_{\mathrm{I}}}=\boldsymbol{e}_{n}^{d_{\mathrm{I}}}$. The linearized equation (3.6) leads to the algebraic system of equations

$$
\underbrace{\left[\mathbf{C}_{\text {lu } n+1, k}^{d_{\mathrm{J}}} \delta_{d_{\mathrm{I}} d_{\mathrm{J}}}+\mathbb{P}^{d_{\mathrm{I}}}: \mathbf{C}_{n+1, k}^{\mathrm{ep}} \mathbb{P}^{d_{\mathrm{J}}^{\mathrm{T}}}\right]}_{\left(\mathbf{A}_{d_{1} d_{\mathrm{J}}}\right)_{n+1, k}} \Delta \boldsymbol{e}_{n+1, k}^{d_{\mathrm{J}}}=\boldsymbol{R}^{d_{\mathrm{I}}}\left(\boldsymbol{e}_{n+1_{k}}^{\mathrm{d}}\right)
$$

involving the same matrix $\boldsymbol{A}_{d_{\mathrm{I}} d_{\mathrm{J}}}$ as in the continuum rate equations but with the damage algorithmic consistent tangent $\mathbf{C}_{\mathrm{lu} n+1_{k}}^{d_{\mathrm{J}}}$ and the elastoplastic algorithmic consistent tangent $\mathbf{C}_{n+1_{k}}^{\mathrm{ep}}$. The final global tangent is obtained exactly as in the continuum case, resulting in the expression

$$
d \boldsymbol{\sigma}_{n+1}=\mathbf{C}_{n+1}^{\mathrm{epd}} d \boldsymbol{\varepsilon}_{n+1}
$$

with 


$$
\mathbf{C}_{n+1}^{\mathrm{epd}}=\mathbf{C}_{n+1}^{\mathrm{ep}}-\sum_{d_{\mathrm{I}}, d_{\mathrm{J}}=1}^{n_{\mathrm{dam}}} \mathbf{C}_{n+1}^{\mathrm{ep}}: \mathbb{P}^{d_{\mathrm{I}}^{\mathrm{T}}}\left(\boldsymbol{A}^{-1}\right)_{d_{\mathrm{I}} d_{J_{n+1}}} \mathbb{P}^{d_{\mathrm{J}}}: \mathbf{C}_{n+1}^{\mathrm{ep}}
$$

with the consistent matrix $\boldsymbol{A}_{d_{1} d_{J_{n+1}}}$ used in the iteration equations (3.8).

The problem then reduces to an independent integration of the elastoplastic model and each of the damage mechanism, that is, the evaluation of stresses (3.5) and (3.4), respectively, with the corresponding updates of the internal variables $\mathscr{I}_{n+1_{k}}^{\mathrm{p}}$ and $\mathscr{I}_{n+1_{k}}^{d_{\mathrm{l}}}$. This integration is accomplished through separate schemes sharing exactly the same structure of standard return mapping algorithms, including their exact closed-form linearization for the algorithmic consistent tangents $\mathbf{C}_{n+1_{k}}^{\mathrm{ep}}$ and $\mathbf{c}_{\mathrm{lu} n+1_{k}}^{d_{1}}$ for each damage mechanism. These integration algorithms are presented in the next two sections.

Remark 3.1. The consideration of a plastic model in the effective stress space $\overline{\boldsymbol{\sigma}}^{\mathrm{d}}:=\boldsymbol{M}^{-1} \boldsymbol{\sigma}^{\mathrm{d}}$, as discussed in Remark 3.3 of Part I, can also be incorporated in this numerical solution strategy after noting that the damage tensor $\boldsymbol{M}$ can also be considered a function of the damage strains $\boldsymbol{e}^{\mathrm{d}}$ through its evolution equation in terms of the evolution of the damage compliance $\mathbf{D}^{\mathrm{d}}$ identified in this work. In this case, after the integration of the damage model, the elastoplastic model can be integrated for the updated damage tensor in the iteration process to enforce the final equilibrium relation (3.3). The linearization in this case collects extra terms due to this dependence, leading to a non-symmetric tangent as in the continuum problem. Further details are omitted at this time.

\subsection{Integration of the elastoplastic model}

The elastoplastic stresses $\boldsymbol{\sigma}_{n+1_{k}}^{\mathrm{ep}}$ in Eq. (3.4) and the corresponding algorithmic consistent tangent $\mathbf{C}_{n+1, k}^{\mathrm{ep}}$ are obtained through a standard return mapping scheme. We note that in the iteration process (3.6) the strains $\varepsilon_{n+1}-\boldsymbol{\varepsilon}_{n+1_{k}}^{\mathrm{d}}$ are fixed for a given iteration, with the aforementioned return mapping scheme returning also the update values of the plastic strains $\varepsilon_{n+1, k}^{\mathrm{p}}$ and plastic internal variables $\mathscr{I}_{n+1, k}^{\mathrm{p}}$. Briefly, this algorithm reads as follows in the general form:

$$
\begin{aligned}
& \text { Define the trial state: } \\
& \boldsymbol{\sigma}_{n+1_{k}}^{\text {eptrial }}=\frac{\partial W^{\mathrm{e}}}{\partial \varepsilon^{\mathrm{e}}}\left(\boldsymbol{\varepsilon}_{n+1}-\boldsymbol{\varepsilon}_{n+1_{k}}^{\mathrm{d}}-\boldsymbol{\varepsilon}_{n}^{\mathrm{p}}\right), \quad \mathscr{I}_{n+1_{k}}^{\text {trial }}=\mathscr{I}_{n}^{\mathrm{p}}, \\
& \operatorname{IF}\left(\phi^{\mathrm{p}}\left(\boldsymbol{\sigma}_{n+1_{k}}^{\mathrm{ep}^{\text {trial }}}, \mathscr{Q}^{\mathrm{p}}\left(\mathscr{I}_{n+1_{k}}^{\mathrm{p}^{\text {trial }}}\right)\right) \leqslant 0\right) \text { THEN } \\
& \boldsymbol{\sigma}_{n+1_{k}}^{\mathrm{e}}=\boldsymbol{\sigma}_{n+1_{k}}^{\mathrm{e}}, \quad \boldsymbol{\varepsilon}_{n+1_{k}}^{\mathrm{p}}=\boldsymbol{\varepsilon}_{n+1_{k}}^{\text {trial }}, \quad \mathscr{I}_{n+1_{k}}^{\mathrm{trial}}=\mathscr{I}_{n+1_{k}}^{\mathrm{p}}, \\
& \text { ELSE } \\
& \text { Solve for } \boldsymbol{\sigma}_{n+1_{k}}^{\mathrm{ep}}, \boldsymbol{\varepsilon}_{n+1_{k}}^{\mathrm{p}} \text { and } \mathscr{I}_{n+1_{k}}^{\mathrm{p}} \text { through a Newton scheme : } \\
& \left\{\begin{array}{l}
\phi^{\mathrm{p}}\left(\boldsymbol{\sigma}_{n+1_{k}}^{\mathrm{ep}}, \mathscr{Q}^{\mathrm{p}}\left(\mathscr{I}_{n+1_{k}}^{\mathrm{p}}\right)\right)=0, \\
\boldsymbol{\varepsilon}_{n+1_{k}}^{\mathrm{p}}=\boldsymbol{\varepsilon}_{n}^{\mathrm{p}}+\Delta \gamma_{n+1_{k}}^{\mathrm{p}} \frac{\partial \phi^{\mathrm{p}}}{\partial \boldsymbol{\sigma}}\left(\boldsymbol{\sigma}_{n+1_{k}}^{\mathrm{ep}}, \mathscr{Q}^{\mathrm{p}}\left(\mathscr{I}_{n+1_{k}}^{\mathrm{p}}\right)\right), \\
\mathscr{I}_{n+1_{k}}^{\mathrm{p}}=\mathscr{I}_{n}^{\mathrm{p}}+\Delta \gamma_{n+1_{k}}^{\mathrm{p}} \frac{\partial \phi^{\mathrm{p}}}{\partial \mathscr{Q}^{\mathrm{p}}}\left(\boldsymbol{\sigma}_{n+1_{k}}^{\mathrm{ep}}, \mathscr{Q}^{\mathrm{p}}\left(\mathscr{I}_{n+1_{k}}^{\mathrm{p}}\right)\right), \\
\boldsymbol{\sigma}_{n+1_{k}}^{\mathrm{ep}}=\frac{\partial W^{\mathrm{e}}}{\partial \boldsymbol{\varepsilon}^{\mathrm{e}}}\left(\boldsymbol{\varepsilon}_{n+1}-\boldsymbol{\varepsilon}_{n+1_{k}}^{\mathrm{d}}-\boldsymbol{\varepsilon}_{n+1_{k}}^{\mathrm{p}}\right) .
\end{array}\right.
\end{aligned}
$$

These equations define the so-called closest point projection scheme and can be exactly linearized in closed form, leading to the so-called algorithmic consistent tangent $\mathbf{C}_{n+1_{k}}^{\mathrm{ep}}$ by the relation

$$
d \boldsymbol{\sigma}_{n+1_{k}}^{\mathrm{ep}}=\mathbf{C}_{n+1_{k}}^{\mathrm{ep}} d\left(\boldsymbol{\varepsilon}_{n+1}-\boldsymbol{\varepsilon}_{n+1_{k}}^{\mathrm{d}}\right) .
$$

For the von Mises yield criterion (2.1) with the isotropic linear elastic response (2.7), Eqs. (3.11) reduce to the well-known radial return algorithm. We refer to Simo and Hughes (1997, p. 124) for details. For completeness, Table 2 includes a summary of these standard equations. 
Table 2

Standard radial return mapping algorithm for the Mises yield criterion (see e.g. Simo and Hughes (1997))

1. Compute trial state: $\quad \sigma_{n+1_{k}}^{\text {eptrial }}=p_{n+1_{k}}^{\mathrm{ep}}+2 \mu^{\mathrm{e}} \operatorname{dev}\left[\boldsymbol{\varepsilon}_{n+1}-\boldsymbol{\varepsilon}_{n+1_{k}}^{\mathrm{d}}-\boldsymbol{\varepsilon}_{n}^{\mathrm{p}}\right], p_{n+1_{k}}^{\mathrm{ep}}=\kappa^{\mathrm{e}} \operatorname{tr}\left[\boldsymbol{\varepsilon}_{n+1}-\boldsymbol{\varepsilon}_{n+1_{k}}^{\mathrm{d}}\right], \quad \alpha_{n+1_{k}}^{\mathrm{p}^{\mathrm{trial}}}=\alpha_{n}^{\mathrm{p}}$.

2. Check consistency of the trial state:

$$
\begin{aligned}
& \operatorname{IF}\left(\phi^{\mathrm{p}}\left(\boldsymbol{\sigma}_{n+1_{k}}^{\text {eptrial }}, q^{\mathrm{p}}\left(\alpha_{n+1_{k}}^{\mathrm{p}^{\text {trial }}}\right)\right)<0\right) \text { THEN } \\
& \boldsymbol{\sigma}_{n+1_{k}}^{\mathrm{ep}}=\boldsymbol{\sigma}_{n+1_{k}}^{\text {etrial }}, \quad \alpha_{n+1_{k}}^{\mathrm{p}}=\alpha_{n+1_{k}}^{\text {trial }}, \quad \mathbf{C}_{n+1_{k}}^{\mathrm{ep}}=\mathbf{C}^{\mathrm{e}} \text { and EXIT } \\
& \text { ELSE (return mapping) ENDIF }
\end{aligned}
$$

3. Return mapping: Solve for $\Delta \gamma_{n+1_{k}}^{\mathrm{p}}>0$ using Newton's method

$$
\phi_{n+1_{k}}^{\mathrm{p}}=\left\|\boldsymbol{s}_{n+1_{k}}^{\text {trial }}\right\|-2 \mu^{\mathrm{e}} \Delta \gamma_{n+1_{k}}^{\mathrm{p}}-\sqrt{\frac{2}{3}}\left[y_{0}^{\mathrm{p}}-q^{\mathrm{p}}\left(\alpha_{n+1_{k}}^{\mathrm{p}}\right)\right]=0
$$

with the updates

$$
\begin{aligned}
& \alpha_{n+1_{k}}^{\mathrm{p}}=\alpha_{n}^{\mathrm{p}}+\sqrt{2 / 3} \Delta \gamma_{n+1_{k}}^{\mathrm{p}}, \quad \boldsymbol{\varepsilon}_{n+1_{k}}^{\mathrm{p}}=\boldsymbol{\varepsilon}_{n}^{\mathrm{p}}+\Delta \gamma_{n+1_{k}}^{\mathrm{p}} \boldsymbol{n}_{\phi_{n+1_{k}}^{\mathrm{p}}}, \boldsymbol{\sigma}_{n+1_{k}}^{\mathrm{ep}}=p_{n+1_{k}}^{\mathrm{ep}}+2 \mu^{\mathrm{e}} \operatorname{dev}\left[\boldsymbol{\varepsilon}_{n+1}-\boldsymbol{\varepsilon}_{n+1_{k}}^{\mathrm{d}}-\boldsymbol{\varepsilon}_{n+1_{k}}^{\mathrm{p}}\right] \\
& \text { and } \boldsymbol{n}_{\phi_{n+1_{k}}^{\mathrm{p}}}^{\mathrm{p}}=\boldsymbol{n}_{\phi_{n+1_{k}}^{\mathrm{p}} \text { trial }}^{\mathrm{p}} . \text { The algorithmic consistent elastoplastic tangent is given by } \\
& \boldsymbol{C}_{n+1_{k}}^{\mathrm{ep}}=\kappa^{\mathrm{e}} \mathbf{1} \otimes \mathbf{1}+2 \mu^{\mathrm{e}} \theta_{n+1_{k}}\left[\boldsymbol{I}-\frac{1}{3} \mathbf{1} \otimes \mathbf{1}\right]-2 \mu^{\mathrm{e}} \bar{\theta}_{n+1_{k}} \boldsymbol{n}_{\phi_{n+1_{k}}^{\mathrm{p}}}^{\mathrm{p}} \otimes \boldsymbol{n}_{\phi_{n+1_{k}}^{\mathrm{p}}}^{\mathrm{p}} \\
& \theta_{n+1_{k}}:=1-\frac{2 \mu^{\mathrm{e}} \Delta \gamma_{n+1_{k}}^{\mathrm{p}}}{\left\|\boldsymbol{s}_{n+1_{k}}^{\mathrm{trial}}\right\|}, \quad \bar{\theta}_{n+1_{k}}:=\frac{1}{1+\frac{K_{n+1_{k}}}{3 \mu^{\mathrm{e}}}}-\left(1-\theta_{n+1_{k}}\right)
\end{aligned}
$$

\subsection{Independent integration of each damage mechanism}

The evaluation of the damage stresses $s_{n+1_{k}}$ for each iteration of the solution process of the residual equations (3.3) is obtained through the constitutive relation (3.5) as

$$
\boldsymbol{s}_{n+1_{k}}^{d_{\mathrm{I}}}=\frac{\partial \widehat{W}^{d_{\mathrm{I}}}}{\partial \boldsymbol{e}^{d_{\mathrm{I}}}}\left(\boldsymbol{e}_{n+1_{k}}^{d_{\mathrm{I}}} ; \mathscr{I}_{n+1_{k}}^{d_{\mathrm{I}}}\right)
$$

for the given current damage strains $\boldsymbol{e}_{n+1_{k}}^{d_{\mathrm{I}}}$ and with the updated internal variables $\mathscr{I}_{n+1_{k}}^{d_{\mathrm{I}}}$ obtained by a backward-Euler approximation of their evolution equations $\left(\dot{J}^{d_{\mathrm{I}}}=\gamma^{d_{\mathrm{I}}} \partial \tilde{\phi}^{d_{\mathrm{I}}} / \partial \mathscr{Q}^{d_{\mathrm{I}}}\right.$ for the associated case). The final system of equations is solved through an operator split following the very same structure of the return mapping algorithm (3.11) employed in the integration of the elastoplastic model. Briefly, these equations read in general form and for each damage mechanism $d_{\mathrm{I}}=1, n_{\mathrm{dam}}$ as

$$
\begin{aligned}
& \text { Define the trial state: } \\
& \boldsymbol{s}_{n+1_{k}}^{d_{\mathrm{I}}^{\text {trial }}}=\frac{\partial W^{d_{\mathrm{I}}}}{\partial e^{d_{\mathrm{I}}}}\left(\boldsymbol{e}_{n+1_{k}}^{d_{\mathrm{I}}}, \mathscr{I}_{n}^{d_{\mathrm{I}}}\right), \quad \mathscr{I}_{n+1_{k}}^{d_{\mathrm{r}}^{\text {trial }}}=\mathscr{I}_{n}^{d_{\mathrm{I}}}, \\
& \operatorname{IF}\left(\tilde{\phi}^{d_{\mathrm{I}}}\left(\mathscr{Q}^{d_{\mathrm{I}}}\left(\boldsymbol{s}_{n+1_{k}}^{d_{\mathrm{I}}^{\text {trial }}}, \mathscr{I}_{n+1_{k}}^{d_{\mathrm{I}}^{\text {trial }}}\right)\right) \leqslant 0\right) \text { THEN } \\
& \boldsymbol{s}_{n+1_{k}}^{d_{\mathrm{I}}}=\boldsymbol{s}_{n+1_{k}}^{d_{\mathrm{I}}^{\text {trial }}}, \quad \mathscr{I}_{n+1_{k}}^{d_{\mathrm{I}}}=\mathscr{I}_{n+1_{k}}^{d_{\mathrm{I}}^{\text {trial }}}, \\
& \text { ELSE } \\
& \text { Solve for } \boldsymbol{s}_{n+1_{k}}^{d_{\mathrm{I}}} \text { and } \mathscr{I}_{n+1_{k}}^{d_{\mathrm{I}}} \text { through a Newton scheme : } \\
& \left\{\begin{array}{l}
\tilde{\phi}^{d_{\mathrm{I}}}\left(\mathscr{Q}^{\mathrm{p}}\left(\boldsymbol{s}_{n+1_{k}}^{d_{\mathrm{I}}}, \mathscr{I}_{n+1_{k}}^{d_{\mathrm{I}}}\right)\right)=0, \\
\mathscr{I}_{n+1_{k}}^{d_{\mathrm{I}}}=\mathscr{I}_{n}^{d_{\mathrm{I}}}+\Delta \gamma_{n+1_{k}}^{d_{\mathrm{I}}} \frac{\partial \tilde{\phi}^{d_{\mathrm{I}}}}{\partial \mathscr{2}^{d_{\mathrm{I}}}}\left(\mathscr{Q}^{\mathrm{p}}\left(\boldsymbol{s}_{n+1_{k}}^{d_{\mathrm{I}}}, \mathscr{I}_{n+1_{k}}^{d_{\mathrm{I}}}\right)\right), \\
\boldsymbol{s}_{n+1_{k}}^{d_{\mathrm{I}}}=\frac{\partial W^{d_{\mathrm{I}}}}{\partial \boldsymbol{e}^{d_{\mathrm{I}}}}\left(\boldsymbol{e}_{n+1_{k}}^{d_{\mathrm{I}}}, \mathscr{I}_{n+1_{k}}^{d_{\mathrm{I}}}\right) .
\end{array}\right.
\end{aligned}
$$


Eq. (3.14) are linearized leading to the algorithmic consistent damage tangent defined by the relation

$$
d \boldsymbol{s}_{n+1_{k}}^{d_{\mathrm{I}}}=\mathbf{c}_{\mathrm{lu}_{n+1_{k}}^{d_{\mathrm{I}}}}^{d_{1}} \boldsymbol{e}_{n+1_{k}}^{d_{\mathrm{I}}}
$$

for each damage mechanism $d_{\mathrm{I}}=1, n_{\mathrm{dam}}$.

Table 3 summarizes the return mapping scheme (3.14) applied to the damage model developed in Section 2. The Newton scheme in the return mapping part reduces to in this case, after some algebraic manipulations, to

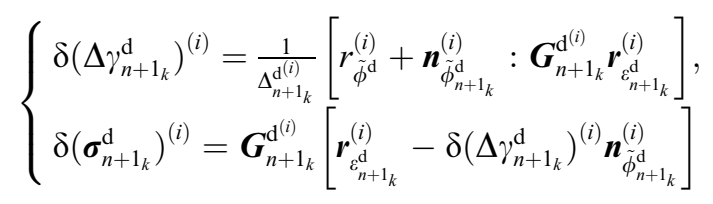

for the local Newton iteration index $(i)$ and

$$
\left\{\begin{array}{l}
\left(\Delta \gamma_{n+1_{k}}^{\mathrm{d}}\right)^{(i+1)}=\left(\Delta \gamma_{n+1_{k}}^{\mathrm{d}}\right)^{(i)}+\delta\left(\Delta \gamma_{n+1_{k}}^{\mathrm{d}}\right)^{(i)}, \\
\left(\boldsymbol{\sigma}_{n+1_{k}}^{\mathrm{d}}\right)^{(i+1)}=\left(\boldsymbol{\sigma}_{n+1_{k}}^{\mathrm{d}}\right)^{(i)}+\delta\left(\boldsymbol{\sigma}_{n+1_{k}}^{\mathrm{d}}\right)^{(i)} .
\end{array}\right.
$$

The residuals $r_{\tilde{\phi}^{\mathrm{d}}}$ and $\boldsymbol{r}_{\varepsilon_{n+1_{k}}^{\mathrm{d}}}$, as well as the tangent matrix $\boldsymbol{G}_{n+1_{k}}^{\mathrm{d}}$ and $\Delta_{n+1_{k}}^{\mathrm{d}^{(i)}}$, are defined Table 3. The final updates for the damage internal variables $\alpha_{n+1_{k}}^{\mathrm{d}}$ and $\mathbf{D}_{n+1_{k}}^{\mathrm{d}}$ are also given there. We note that in the case of $\mathbf{D}_{n+1_{k}}^{\mathrm{d}}$ this is a rank-one update, thus leading to the closed-form expression for the secant tangent

$$
\mathbf{C}_{n+1_{k}}^{\mathrm{d}}=\mathbf{C}_{n}^{\mathrm{d}}-\frac{\Delta \gamma_{n+1_{k}}^{\mathrm{d}}}{\Xi_{n+1_{k}}^{\mathrm{d}}} \mathbf{C}_{n}^{\mathrm{d}} \boldsymbol{n}_{\tilde{\phi}_{n+1_{k}}^{\mathrm{d}}} \otimes \mathbf{C}_{n}^{\mathrm{d}} \boldsymbol{n}_{\tilde{\phi}_{n+1_{k}}^{\mathrm{d}}},
$$

where

$$
\Xi_{n+1_{k}}^{\mathrm{d}}=\boldsymbol{n}_{\tilde{\phi}_{n+1_{k}}^{\mathrm{d}}}: \boldsymbol{\sigma}_{n+1_{k}}^{\mathrm{d}}+\Delta \gamma_{n+1_{k}}^{\mathrm{d}} \boldsymbol{n}_{\tilde{\phi}_{n+1_{k}}^{\mathrm{d}}}: \mathbf{C}_{n}^{\mathrm{d}} \boldsymbol{n}_{\tilde{\phi}_{n+1_{k}}^{\mathrm{d}}}>0,
$$

a strictly positive denominator since $\Delta \gamma_{n+1_{k}}^{\mathrm{d}} \geqslant 0$ by the Kuhn-Tucker conditions, $\boldsymbol{n}_{\tilde{\phi}_{n+1_{k}}^{\mathrm{d}}}: \boldsymbol{\sigma}_{n+1_{k}}^{\mathrm{d}}>0$ by the assumed convexity of the elastic damage domain, and the $\boldsymbol{n}_{\tilde{\phi}_{n+1_{k}}^{\mathrm{d}}}: \mathbf{C}_{n_{k}}^{\mathrm{d}} \boldsymbol{n}_{\tilde{\phi}_{n+1_{k}}^{\mathrm{d}}}>0$ by the positive definiteness of the secant stiffness (or its semi-definiteness for a purely volumetric damage evolution, following the discussion in Remark 2.1). We also note that the same tangent terms used in the Newton process (3.16) lead to the closed-form expression of the algorithmic consistent tangent $\mathbf{c}_{\mathrm{lu}_{n+1_{k}}}^{\mathrm{d}}$ as presented in the return mapping algorithm (Remark 3.2).

Remark 3.2. (1) Referring to the damage return mapping summarized below, we note that $\mathbf{D}_{n}^{\mathrm{d}}=0$ before the damage mechanism is activated (note that after activation no singularity appears in subsequent time steps as discussed in Eq. (3.18)). To activate the damage mechanism, the trial stresses $\boldsymbol{\sigma}^{\mathrm{d}^{\text {trial }}}$ can be alternatively defined as $\boldsymbol{\sigma}^{\mathrm{ep}}$, that is, the stresses obtained in the elastoplastic model, avoiding the inversion of $\mathbf{D}_{n}^{\mathrm{d}}$, and gives directly the final stress if the damage mechanism is not activated when checking damage consistency. If the damage mechanism is activated in the process (i.e. damage consistency is violated by the trial state $\boldsymbol{\sigma}^{\mathrm{ep}}$ ), the damage return mapping proceeds as indicated in Table 3. The tangent matrix involved in solving the non-linear system in the Newton's method Eq. (3.16) involves the calculation of the inverse in matrix $\boldsymbol{G}^{\mathrm{d}}$ as defined above, which is in general non-singular when starting from $\Delta \gamma^{\mathrm{d}} \neq 0$. If a purely volumetric damage evolution is activated leading to a singularity, the reduced form of Remark 2.1 can be used leading to invertible damage relations.

(2) Continuing with this purely volumetric limit (that is, $a=0$ in Eq. (2.8)), the return mapping algorithm reduces in this case to the evaluation of the given hardening/softening law in terms $e_{\mathrm{v}_{n+1} k_{k}}^{\mathrm{d}}=\operatorname{tr}\left[\boldsymbol{\varepsilon}_{n+1_{k}}^{\mathrm{d}}\right]$ of Remark 2.1.2, i.e., 
Table 3

Return mapping algorithm for the quadratic damage model of Section 2, for $r_{\text {given }}^{\mathrm{a}}$ damage strain $\boldsymbol{\varepsilon}_{n+1_{k}}^{\mathrm{d}}$ in iteration $k$ of the solution process of Eq. (3.3) in increment $\left[t_{n}, t_{n+1}\right]$

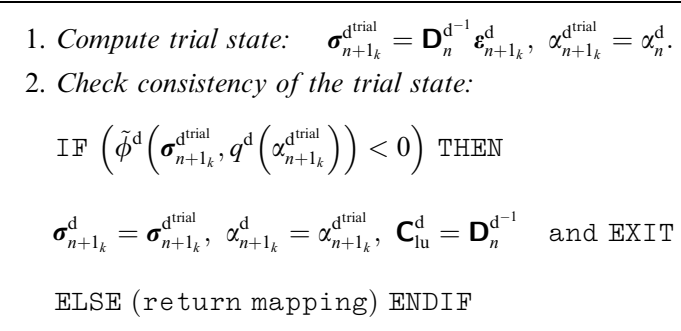

3. Return mapping: Solve for $\Delta \gamma_{n+1_{k}}^{\mathrm{d}}>0$ and $\boldsymbol{\sigma}_{n+1_{k}}^{\mathrm{d}}$ through a Newton scheme

$$
\left.\begin{array}{l}
r_{\tilde{\phi}^{\mathrm{d}}}=\phi^{\mathrm{d}}\left(\boldsymbol{\sigma}_{n+1_{k}}^{\mathrm{d}}, q^{\mathrm{d}}\left(\alpha_{n+1_{k}}^{\mathrm{d}}\right)\right)=0, \\
\boldsymbol{r}_{\epsilon^{\mathrm{d}}}:=\boldsymbol{\varepsilon}_{n+1_{k}}^{\mathrm{d}}-\mathbf{D}_{n}^{\mathrm{d}} \boldsymbol{\sigma}_{n+1_{k}}^{d_{1}}-\Delta \gamma_{n+1_{k}}^{\mathrm{d}} \boldsymbol{n}_{\tilde{\phi}_{n+1_{k}}^{\mathrm{d}}}=0
\end{array}\right\}
$$

with the update formulae

$$
\begin{aligned}
& \alpha_{n+1_{k}}^{\mathrm{d}}=\alpha_{n}^{\mathrm{d}}+\Delta \gamma_{n+1_{k}}^{\mathrm{d}}, \\
& \mathbf{D}_{n+1_{k}}^{\mathrm{d}}=\mathbf{D}_{n}^{\mathrm{d}}+\Delta \gamma_{n+1_{k}}^{\mathrm{d}} \frac{\boldsymbol{n}_{\phi_{n+1_{k}}^{\mathrm{d}}} \otimes \boldsymbol{n}_{\tilde{\phi}_{n+1_{k}}^{\mathrm{d}}}}{\boldsymbol{n}_{\tilde{\phi}_{n+1_{k}}^{\mathrm{d}}}: \boldsymbol{\sigma}_{n+1_{k}}},
\end{aligned}
$$

and the symmetric closed-form algorithmic consistent tangent

$$
\begin{aligned}
& \mathbf{C}_{\mathrm{lu}_{n+1_{k}}}^{\mathrm{d}}=\boldsymbol{G}_{n+1_{k}}^{\mathrm{d}}-\frac{1}{\Delta_{n+1_{k}}^{\mathrm{d}}} \boldsymbol{G}_{n+1_{k}}^{\mathrm{d}} \boldsymbol{n}_{\tilde{\phi}_{n+1_{k}}^{\mathrm{d}}} \otimes \boldsymbol{G}_{n+1_{k}}^{\mathrm{d}} \boldsymbol{n}_{\tilde{\phi}_{n+1_{k}}^{\mathrm{d}}} \\
& \text { for } \boldsymbol{n}_{\tilde{\phi}_{n+1_{k}}^{\mathrm{d}}}=\left.\frac{\partial \tilde{\phi}^{\mathrm{d}}}{\partial \boldsymbol{\sigma}}\right|_{n+1_{k}}, \boldsymbol{B}_{n+1_{k}}^{\mathrm{d}}=\left.\frac{\partial \mathbf{n}_{\tilde{\phi}_{\mathrm{d}}}}{\partial \boldsymbol{\sigma}}\right|_{n+1_{k}}, \boldsymbol{G}_{n+1_{k}}^{\mathrm{d}}=\left[\mathbf{D}_{n}^{\mathrm{d}}+\Delta \gamma_{n+1_{k}}^{\mathrm{d}} \boldsymbol{B}_{n+1_{k}}^{\mathrm{d}}\right]^{-1} \text {, and } \Delta_{n+1_{k}}^{\mathrm{d}}:=\boldsymbol{n}_{\tilde{\phi}_{n+1_{k}}^{\mathrm{d}}}: \boldsymbol{G}_{n+1_{k}}^{\mathrm{d}} \boldsymbol{n}_{\tilde{\phi}_{n+1_{k}}} \quad+K_{n+1_{k}}^{\mathrm{d}} .
\end{aligned}
$$

$$
p_{n+1_{k}}^{\mathrm{d}}= \begin{cases}\mathbf{c}_{n}^{\mathrm{d}} e_{\mathrm{v}_{n+1_{k}}}^{\mathrm{d}} & \text { if } \quad 0<e_{\mathrm{v}_{n+1_{k}}}^{\mathrm{d}} \leqslant \max _{t \leqslant t_{n}}\left\{e_{\mathrm{v}_{t}}^{\mathrm{d}}\right\} \quad \text { (unloading), } \\ \hat{p}\left(e_{\mathrm{v}_{n+1_{k}}}^{\mathrm{d}}\right) & \text { if } \quad e_{\mathrm{v}_{n+1}}^{\mathrm{d}}>\max _{t \leqslant t_{n}}\left\{e_{\mathrm{v}_{t}}^{\mathrm{d}}\right\} \quad \text { (loading) }\end{cases}
$$

with $p_{n+1_{k}}^{\mathrm{d}}=\rho_{\mathrm{p}} e_{\mathrm{v}_{n+1_{k}}}^{\mathrm{d}}$ for $e_{\mathrm{v}_{n+1_{k}}}^{\mathrm{d}} \leqslant 0$ to model void closing through penalization see (3) below. We also have the update $\mathbf{c}_{\mathrm{v}_{n+1}}^{\mathrm{d}}=p_{n+1_{k}}^{\mathrm{d}} / e_{\mathrm{v}_{n+1_{k}}}^{\mathrm{d}}$ for the damage secant stiffness $\left(\mathbf{d}_{\mathrm{v}_{n+1_{k}}}^{\mathrm{d}}=\mathbf{c}_{\mathrm{v}_{n+1} 1_{k}}^{\mathrm{d}-1}\right)$, with the damage algorithmic consistent tangent simply given in this case as

$$
\mathbf{c}_{\mathrm{lu} n+1_{k}}^{\mathrm{d}}= \begin{cases}\mathbf{c}_{n}^{\mathrm{d}} & (\text { unloading }), \\ \hat{p}^{\prime}\left(e_{\mathrm{v}_{n+1_{k}}}^{\mathrm{d}}\right) & (\text { loading })\end{cases}
$$

in the context of the volumetric formulation of Remark 2.1 and the general discrete equations in reduced form of Section 3.1 .

(3) The numerical activation of the damage mechanism presented above (see 1) can also be easily and efficiently accomplished by penalization. That is, we simply initialize $\mathbf{D}_{0}^{\mathrm{d}}=1 / \rho_{\mathrm{p}} \square\left(\right.$ or, equivalently, $\left.\mathbf{C}_{0}^{\mathrm{d}}=\rho_{\mathrm{p}} \square\right)$ for a large penalty parameter $\rho_{\mathrm{p}}$. Values $\rho_{\mathrm{p}} \sim 10^{8} \mu^{\mathrm{e}}$ have been used in the numerical simulations of Section 4.

(4) Similarly, the penalty version presented in Remark 2.2(2) of the unilateral closing/opening of voids is used (with the same value of the penalty parameter $\rho_{\mathrm{p}}$ as in the previous item) to avoid a treatment similar to the one presented in (1) above to deal with the singularity of expressions like Eq. (2.48). This is easily achieved by adding the penalty term in Eq. (2.50) to the residual $\boldsymbol{r}_{\epsilon^{\mathrm{d}}}$ and proceeding with its consistent linearization; details are omitted. 
(5) The inviscid return mapping algorithm given above can be readily extended to the viscous Perzyna regularization defined by the viscous relation

$$
\gamma^{\mathrm{d}}=\frac{\left\langle\tilde{\phi}^{\mathrm{d}}\right\rangle}{\eta^{\mathrm{d}}}
$$

replacing the damage Kuhn-Tucker conditions (2.13) and consistency condition (2.14). The integration algorithm for this case is easily obtained by rewriting the consistency residual $r_{\tilde{\phi}^{\mathrm{d}}}$ as

$$
r_{\tilde{\phi}^{\mathrm{d}}}=\phi^{\mathrm{d}}\left(\boldsymbol{\sigma}_{n+1_{k}}^{\mathrm{d}}, q^{\mathrm{d}}\left(\alpha_{n+1_{k}}^{\mathrm{d}}\right)\right)-\eta^{\mathrm{d}} \frac{\Delta \gamma_{n+1_{k}}^{\mathrm{d}}}{\Delta t}
$$

for the time increment $\Delta t$ and the damage viscosity $\eta^{\mathrm{d}}$. A linear viscous model in terms of the linear viscosity parameter $\eta^{\mathrm{d}}$ is assumed in Eq. (3.22) for simplicity; extensions to non-linear relations can be easily accommodated. The tangent relations in the return mapping algorithm above apply entirely with the only change

$$
K_{n+1_{k}}^{\mathrm{d}} \rightarrow K_{n+1_{k}}^{\mathrm{d}}+\frac{\eta^{\mathrm{d}}}{\Delta t}
$$

for the hardening/softening modulus.

\section{Representative numerical simulations}

We present in this section several numerical simulations that illustrate the main features of the plastic damage model developed in Section 2. More specifically, Section 4.1 considers two homogeneous strain tests and Section 4.2 the tension test of a perforated strip.

\subsection{Homogeneous strain tests}

We consider two tests problems involving an imposed strain history path. The pressure dependent damage surface (2.8) is considered for both tests, with a softening law defined as described in Remark 2.1(2) by the exponential relation

$$
\hat{p}\left(e_{\mathrm{v}}^{\mathrm{d}}\right)=\omega_{0}^{\mathrm{d}} \exp \left[\frac{H^{\mathrm{d}}}{\omega_{0}^{\mathrm{d}}} e_{\mathrm{v}}^{\mathrm{d}}\right]
$$

in terms of the intermediate internal variable $e_{\mathrm{v}}^{\mathrm{d}}$. The von Mises yield criterion (2.1) is assumed in combination of the saturation isotropic hardening law

$$
y^{\mathrm{p}}\left(\alpha^{\mathrm{p}}\right)=y_{0}^{\mathrm{p}}+\left(y_{\infty}^{\mathrm{p}}-y_{0}^{\mathrm{p}}\right)\left(1-\exp \left[-\delta^{\mathrm{p}} \alpha^{\mathrm{p}}\right]\right) .
$$

The assumed material parameters are summarized in Table 4.

In Test \#1, the cyclic strain history on $\varepsilon_{x x}$ depicted in Fig. 4 is imposed, with all other components of the strain tensor vanishing. More specifically, we impose the strain rates

Table 4

Homogeneous strain tests - material parameters

\begin{tabular}{lll} 
Initial Young's modulus & $E^{\mathrm{e}}$ & 2.0 \\
Initial Poisson's ratio & $v^{\mathrm{e}}$ & 0.3 \\
Initial yield limit & $y_{0}^{\mathrm{p}}$ & 0.25 \\
Saturation yield limit & $y_{\infty}^{\mathrm{p}}$ & 0.8 \\
Saturation plastic hardening exponent & $\delta^{\mathrm{p}}$ & 5.0 \\
Initial damage threshold & $\tilde{\omega}_{0}^{\mathrm{d}}$ & 1.0 \\
Exponential damage softening modulus & $H^{\mathrm{d}}$ & -0.5 \\
Damage surface parameter & $a$ & 0.5 \\
\hline
\end{tabular}




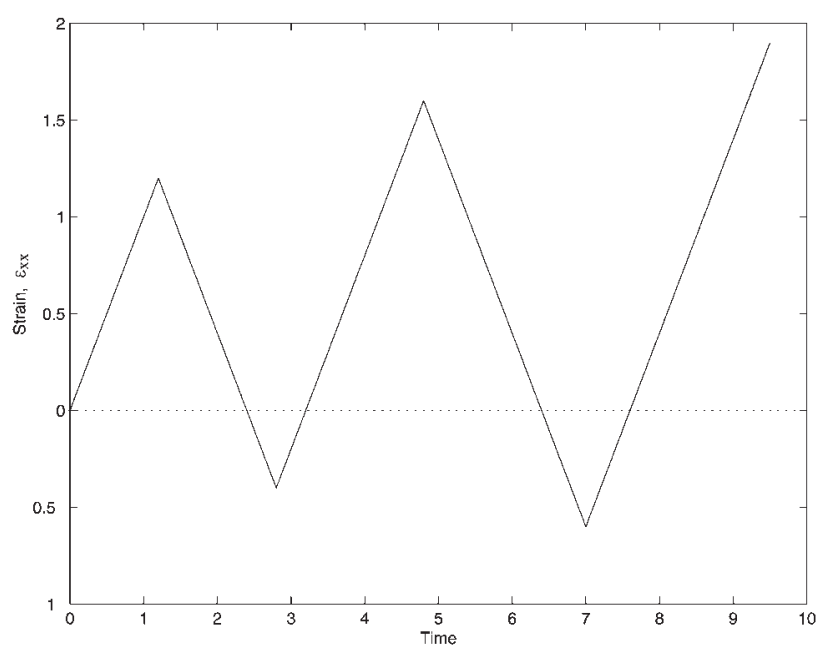

Fig. 4. Homogeneous strain test problems - imposed strain $\varepsilon_{x x}$ versus time.

$$
\dot{\varepsilon}_{x x}=\left\{\begin{array}{rc}
1 & 0<t<1.2 \\
-1 & 1.2<t<2.8 \\
1 & 2.8<t<4.8 \\
-1 & 4.8<t<7.0 \\
1 & 7.0<t<9.5
\end{array}\right.
$$

with $t$ denoting the time variable. A constant time step of $\Delta t=0.01$ is considered in the simulations. This monotonic strain path leads to a tridimensional state of stress, with the principal strains and stress coinciding with the Cartesian axes. Fig. 5 depicts the solution obtained for this test. The evolutions of the axial stress $\sigma_{x x}$ and the hydrostatic pressure $p$ are depicted versus the imposed axial strain $\varepsilon_{x x}$. We also include a representation of the stress path for the first strain cycle $(t \in[0,4.45])$ in the $p-\sqrt{J_{2}}$ plane, for the second invariant of the deviatoric stress $J_{2}=\|\boldsymbol{s}\|^{2} / 2$. The damage surface is also depicted every five time steps.

The different features of the coupled plastic-damage model proposed herein appear in this solution. For example, concentrating on the first strain cycle, we observe after an initial elastic and plastic phases, a fully coupled plastic damage phase. This phase is characterized by the softening response in the pressure $p$ and axial stress $\sigma_{x x}$ plots. Note that the assumed damage softening law (4.1) is accompanied with a hardening plastic response as illustrated in the evolution of the $J_{2}$ stress invariant in the stress path $p-\sqrt{J_{2}}$. This phase is followed by a (damaged) unloading, reaching a level of strain where the unilateral closing of voids is reached. As can be observed in the $p-\varepsilon_{x x}$ plot or the stress path plot, this closing stage is reached even for a positive pressure $p>0$. We note again that the constraint is imposed directly on the damage strains $\operatorname{tr}\left[\boldsymbol{\varepsilon}^{\mathrm{d}}\right] \geqslant 0$. The change in stiffness can be observed in all the three plots of Fig. 5. In particular, we observe that the pressure evolution recovers the elastic stiffness given by the elastic bulk modulus $\kappa^{\mathrm{e}}=E^{\mathrm{e}} /$ $3\left(1-2 v^{\mathrm{e}}\right)$, as discussed after Eq. (2.40). The continuity of the stress response noted in Remark 2.2(3) can be observed in these plots. Under continued unloading, plastic yielding occurs in compression. After reversing once more the applied strain, the material unloads elastically, reaching a stress level where voids open. A sudden degradation of the stiffness can be observed at this point. The test continues, observing the added degradation due to damage and the accumulation of plastic strain.

Table 5 illustrates the performance of the proposed integration scheme. The evolution of the residual Euclidean norm $\left\|\boldsymbol{R}^{\mathrm{d}}\right\|$ in the Newton-iteration process to impose the equilibrium between the elastoplastic 

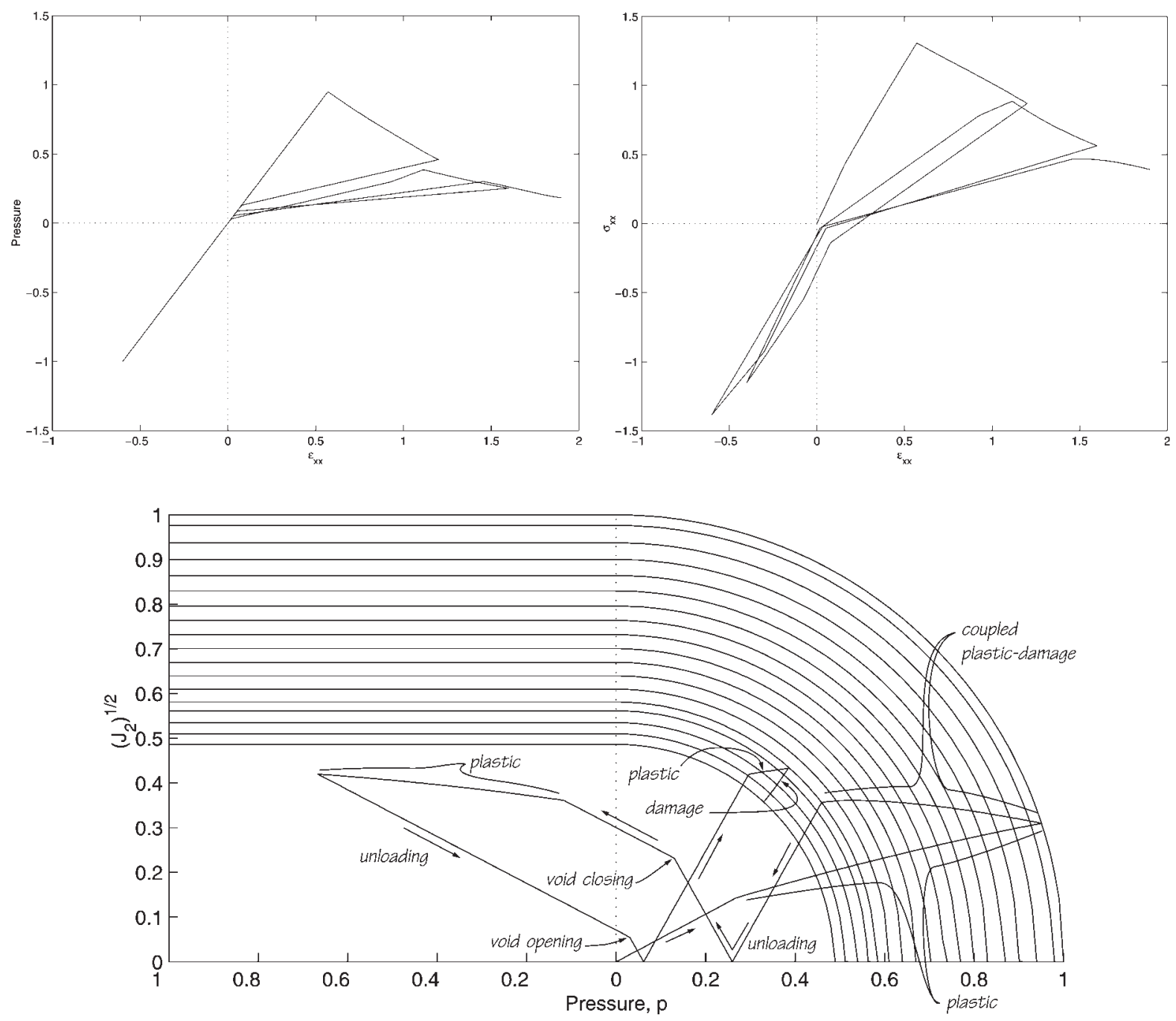

Fig. 5. Test \#1: Monotonic strain path - pressure and stress $\sigma_{x x}$ versus imposed strain $\varepsilon_{x x}$; stress path in the $p-\sqrt{J_{2}}=\|\boldsymbol{s}\| / \sqrt{2}$ plane.

Table 5

Test \#1: typical convergence rates

\begin{tabular}{ll}
\hline Elastoplastic/damage equilibrium $^{\mathrm{a}}$ & Damage mechanism local iteration $^{\mathrm{b}}$ \\
\hline $0.292001 \mathrm{E}-01$ & $0.530605 \mathrm{E}+00$ \\
$0.267391 \mathrm{E}-01$ & $0.137069 \mathrm{E}+00$ \\
$0.149596 \mathrm{E}-01$ & $0.372367 \mathrm{E}-01$ \\
$0.104282 \mathrm{E}-03$ & $0.799946 \mathrm{E}-02$ \\
$0.301675 \mathrm{E}-06$ & $0.301884 \mathrm{E}-03$ \\
$0.696459 \mathrm{E}-13$ & $0.192482 \mathrm{E}-04$ \\
& $0.437543 \mathrm{E}-08$ \\
& $0.742203 \mathrm{E}-16$ \\
\hline
\end{tabular}

${ }^{a}$ Typical convergence rate for the Newton iterative scheme imposing the elastoplastic-damage equilibrium relation (3.3).

${ }^{\mathrm{b}}$ Typical convergence rate for the Newton iterative scheme imposing the consistency relations for the local damage mechanism. 
stresses and the damage stresses (that is, while solving the residual equations (3.3)) is shown in the left column, while the right column includes the evolution of the Euclidean norm $\|\boldsymbol{r}\|^{2}=\left\|\boldsymbol{r}_{\varepsilon^{\mathrm{d}}}\right\|^{2}+\left\|r_{\phi^{\mathrm{d}}}\right\|^{2}$ (even though, both terms are dimensionally different) of the local residual in the iteration, imposing damage consistency (3.16). The second-order convergence in both iterative processes, a direct consequence of the use of the closed-form consistent linearization of the equations as described in Section 3 is verified.

In Test \#2, we apply the same cyclic history on the $\varepsilon_{x x}$. The only difference is that a shear strain is applied during the first loading phase with

$$
\dot{\varepsilon}_{x y}=1 \text { for } 0<t<1.2 \text {, }
$$

keeping this strain constant $\left(\varepsilon_{x y}=1.2\right)$ during the rest of the test. Fig. 6 depicts the solution obtained in this case, showing the evolution of the axial stress $\sigma_{x x}$ versus the imposed axial strain $\varepsilon_{x x}$ as well as the stress path
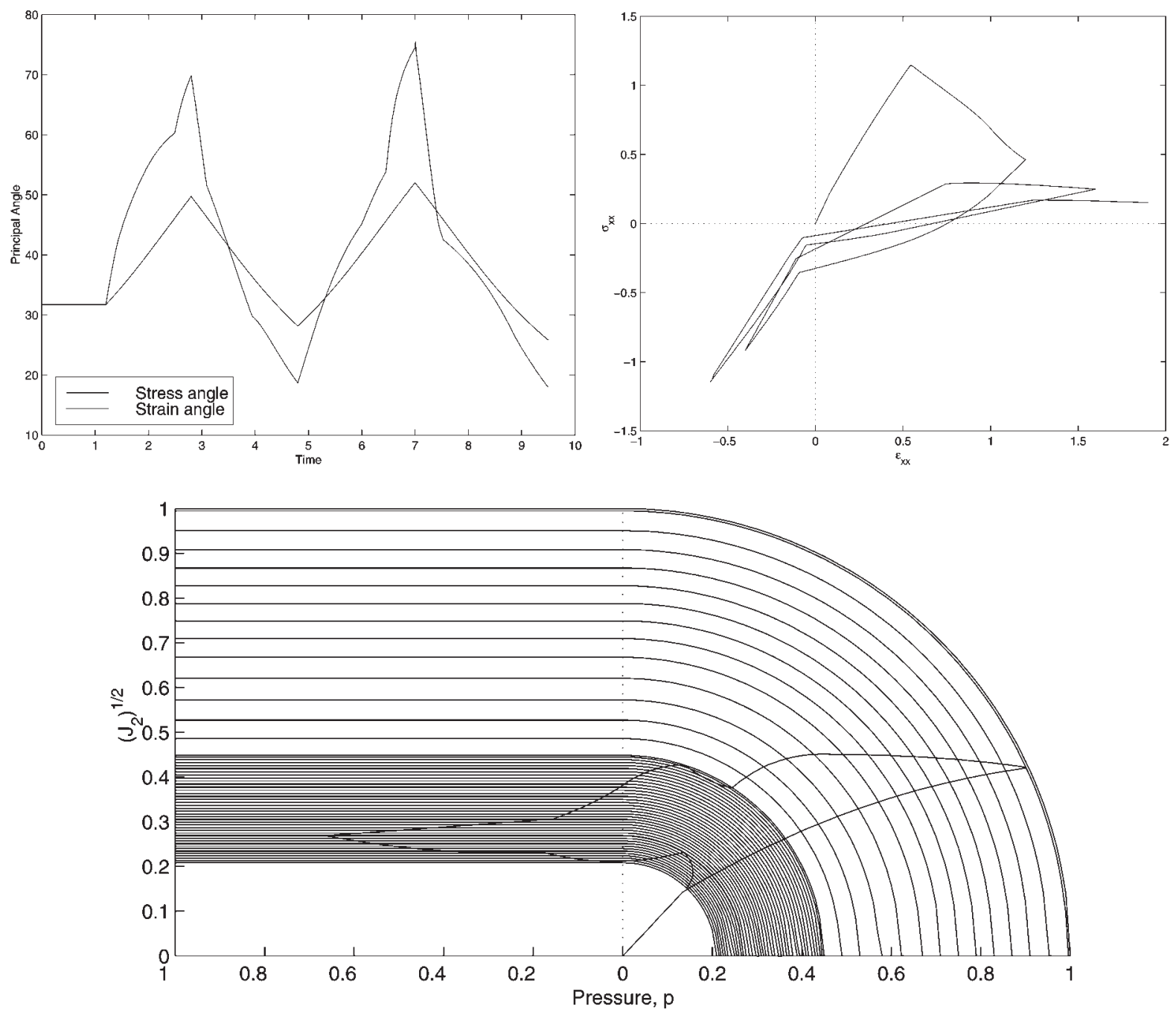

Fig. 6. Test \#2: non-monotonic strain path - principal strain and stress angles, and stress $\sigma_{x x}$ versus imposed strain $\varepsilon_{x x}$; stress path in the $p-\sqrt{J_{2}}=\|\boldsymbol{s}\| / \sqrt{2}$ plane. 
$p-\sqrt{J_{2}}$. We have also included the evolution of the principle angles of stress and strain versus time. This plot allows us to illustrate the induced anisotropy introduced in the material response by both the plastic and damage components of the deformation in this non-monotonic strain path. The evolution of the axial stress $\sigma_{x x}$ shows similar features as the ones discussed in the previous test, with the changing of stiffness due to void closing/opening, as well as the accumulation of plastic strain. A more complex stress path can be observed in this case.

\subsection{Perforated strip problem}

We consider in this section the benchmark problem of the tension test of a perforated strip, as is usually considered in the evaluation of elastoplastic models (Simo and Hughes, 1997). The geometric definition of the specimen is depicted in Fig. 7. A rectangular $20 \times 36$ strip with a circular cavity of radius 5 is stretched axially in plane strain. Due to the symmetry in the problem, only one quarter of the specimen is considered in the numerical simulations with the appropriate symmetry boundary conditions along its borders. The quarter specimen is discretized with 200 Q1/E4 enhanced elements as presented by Simo and Rifai (1990).

The von Mises yield criterion (2.1) is again assumed with a linear isotropic hardening law of modulus $K^{\mathrm{p}}=1.0$ in this case. To illustrate the use of the reduced damage formulation, the pressure dependent damage surface (2.8) is considered with $a=0$, leading to the purely volumetric model described in Remark 2.1. We note the simplified numerical implementation of this reduced case, as discussed in Remark 3.2(2).

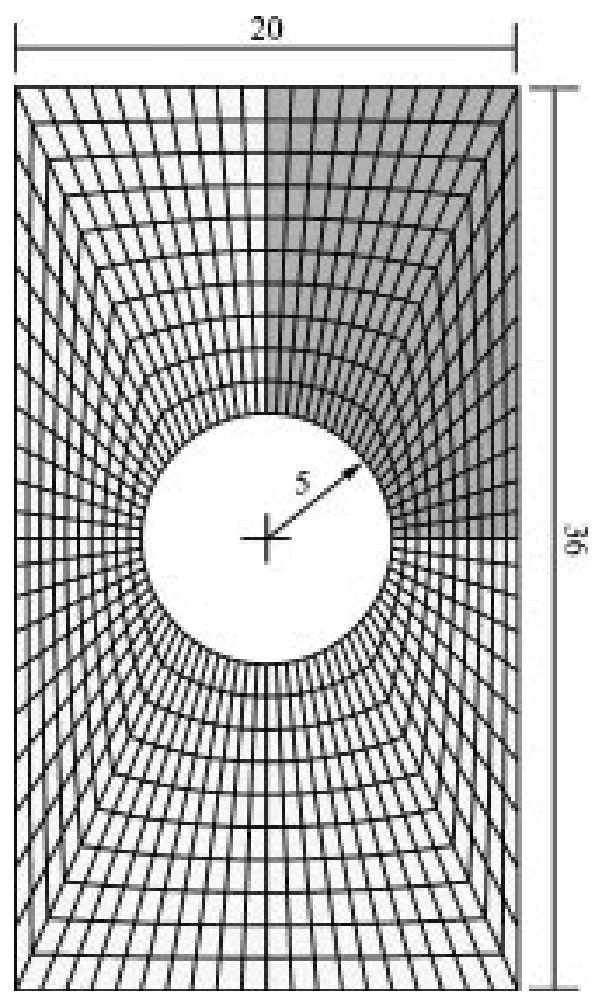

Fig. 7. Perforated strip: problem definition - by symmetry only a quarter of the specimen is discretized and solved for. 
Table 6

Perforated strip problem - material properties

\begin{tabular}{llc}
\hline Young's modulus & $E^{\mathrm{e}}$ & 70.0 \\
Poisson's ratio & $v^{\mathrm{e}}$ & 0.3 \\
Yield limit & $y_{0}^{\mathrm{p}}$ & 0.2 \\
Linear plastic hardening modulus & $K^{\mathrm{p}}$ & 1.0 \\
Initial damage threshold & $\omega_{0}^{\mathrm{d}}$ & 0.1 \\
Exponential damage softening modulus & $H^{\mathrm{d}}$ & -2.4 \\
Damage surface parameter & $a$ & 0.0 \\
\hline
\end{tabular}

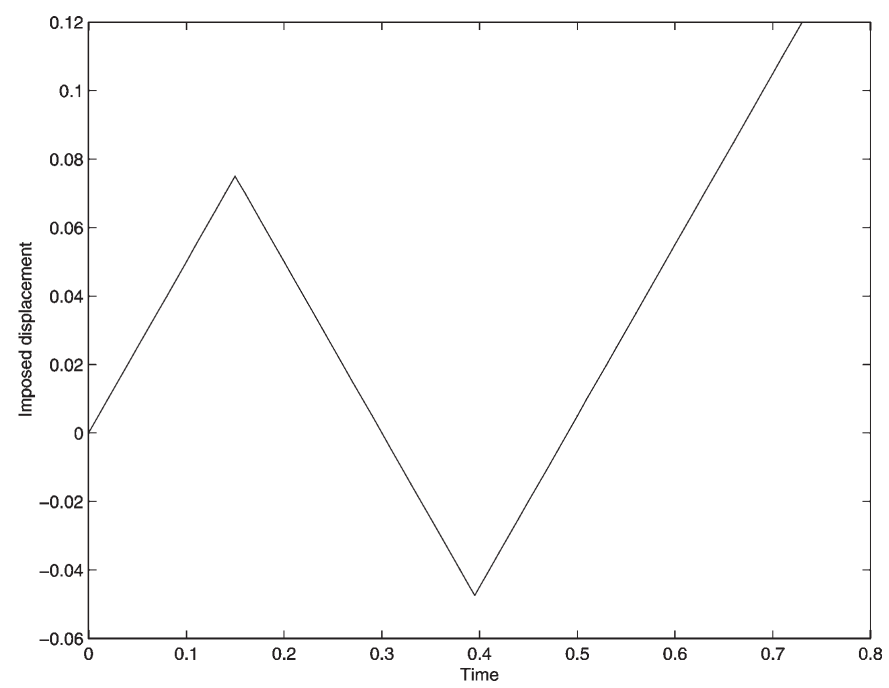

Fig. 8. Perforated strip: imposed displacement history.

The same exponential softening relation (4.1) in terms of the volumetric damage strain $e_{\mathrm{v}}^{\mathrm{d}}=\operatorname{tr}\left[\varepsilon^{\mathrm{d}}\right]$ as in the homogeneous tests of the previous section is considered. The assumed material parameters are summarized in Table 4. We consider simulations with the coupled plastic-damage model and with plasticity only. The latter case is obtained by simply setting the damage threshold $\omega_{0}^{\mathrm{d}}$ to a very large value (Table 6).

The simulations are run with an imposed displacement $\bar{u}$ at the top, following the cyclic history shown in Fig. 8 to evaluate the damage in the material. A constant time step of $\Delta t=0.01$ is employed. Fig. 9 shows the solutions obtained for the purely plastic and the plastic-damage simulations. The distribution of the equivalent plastic strain $\alpha^{\mathrm{p}}$ is depicted for both cases superposed to the deformed configuration. The computed load-displacement curves are also included.

The differences between the plastic and plastic-damage solutions are apparent. The damaged stiffness in unloading can be observed in the latter. We can also note the sudden change of stiffness in the unloading phase due to the closing of voids (and similarly in reloading due to void opening). The different pattern of distribution of the equivalent plastic strain is also apparent. We observe the classical $45^{\circ}$ banded pattern in the plastic solution, with a more brittle type (mode I type) when the volumetric damage is considered. Fig. 10 shows the distribution of the volumetric damage strain, a measure of the void fraction created in the material due to damage; see Eq. (2.35). The different shape of the deformed cavity for the two cases is to be noted. 

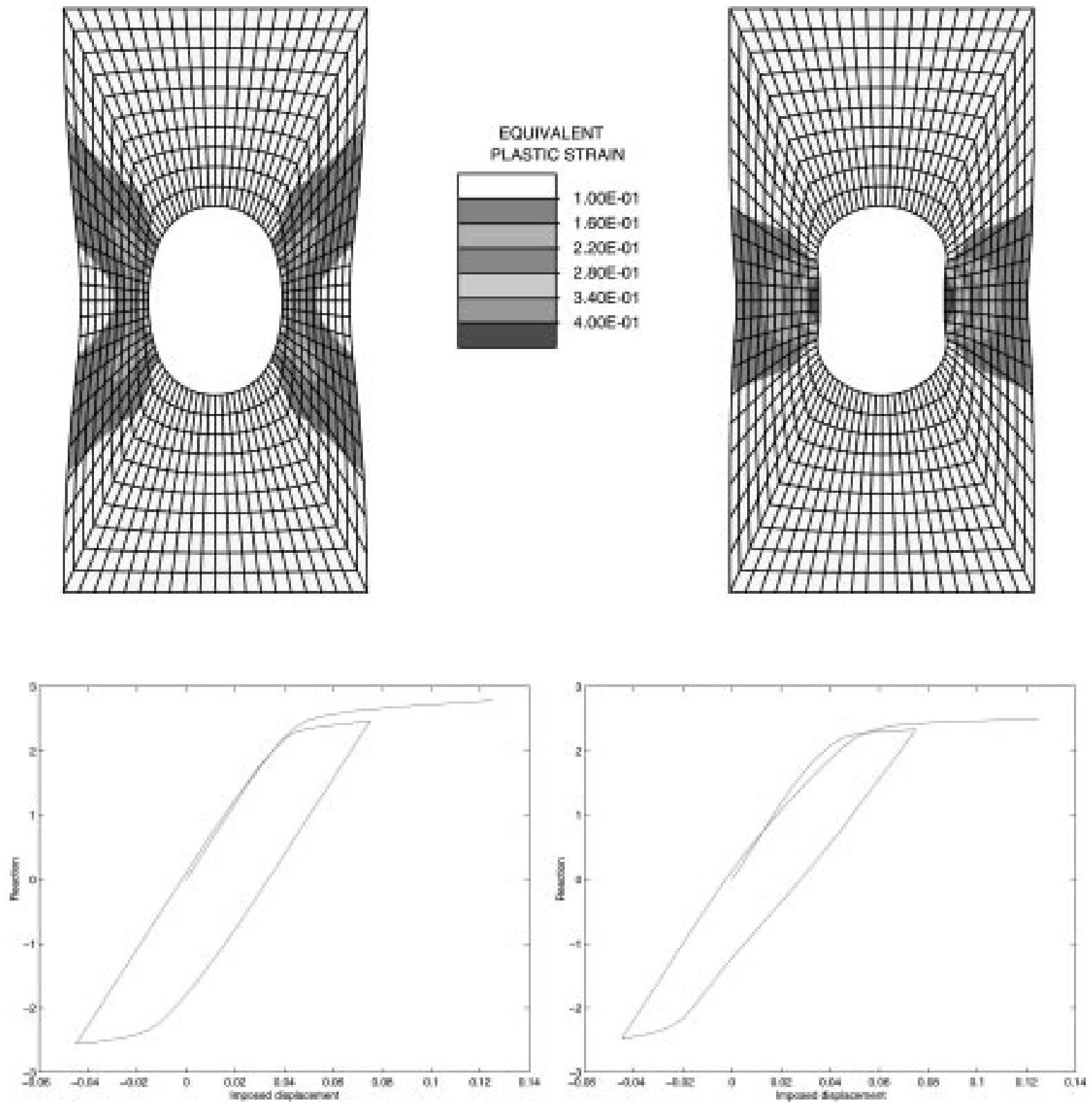

Fig. 9. Perforated strip: comparison of the solutions obtained with the $J_{2}$ elastoplastic model (left) and the considered elastoplasticdamage model (right): distributions of the equivalent plastic strain (at $\bar{u}=1.255$ ) and load-displacement curves.

\section{Concluding remarks}

We have presented in this paper the application of a general framework of continuum damage models to the numerical simulation of the damage and plasticity in porous metals. To this purpose, we have developed a new simple plastic damage model. The proposed model is based on a pressure dependent damage surface, 

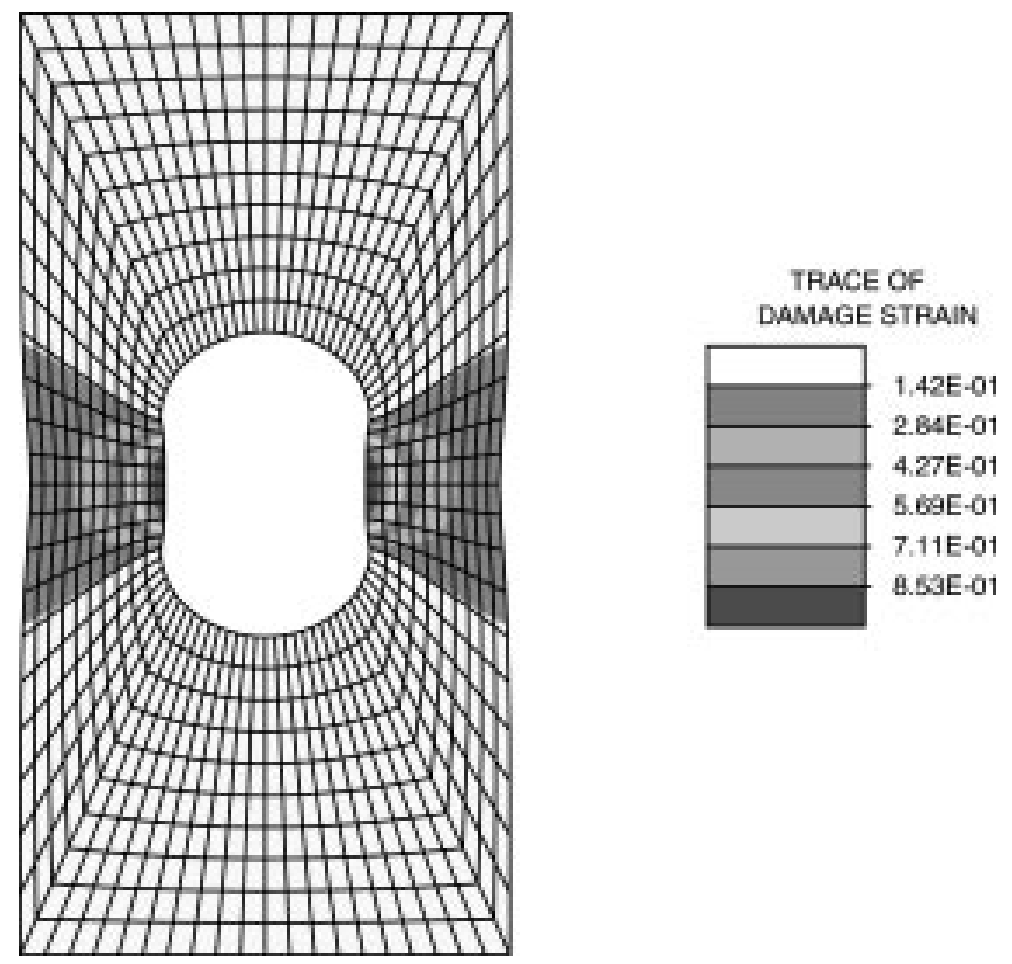

Fig. 10. Perforated strip: distribution of volumetric damage strain $e_{\mathrm{v}}^{\mathrm{d}}:=\operatorname{tr}\left[\boldsymbol{\varepsilon}^{\mathrm{d}}\right]$ at $\bar{u}=1.255$ for the elastoplastic-damage solution.

controlling the degradation of the stiffness of the material, with the permanent plastic strain controlled by the classical von Mises criterion. The proposed damage criterion consists of a combination of the distorsional and volumetric strain energies in the material, the latter part considered in tension only. The evolution laws for the internal damage variables, namely, the damage compliance and a scalar variable modeling the irreversible cohesive opening of voids in the metallic matrix, have been formulated in an associated form from the principle of maximum damage dissipation. Furthermore, the unilateral damage effects associated to the closing/opening of voids have also been incorporated through the imposition of the unilateral constraint of non-negative void fraction.

The resulting model incorporated in a very modular fashion the damage and plastic effects in solids, and most importantly in a highly physically motivated way. In addition, these ideas translate directly in the numerical treatment of the resulting models. We have developed in this paper numerical integration schemes that show also a very convenient modularity in the treatment of the different responses in the material. The main idea to accomplish this modularity is maintaining the damage strains as the driving variables in the numerical implementation. In this way, we have presented a return mapping algorithm for the integration of the proposed damage model, sharing remarkably the exact same structure as the classical return mapping employed in the integration of the plastic part of the model. The final numerical implementation then involves a series of independent routines providing the stresses, updates of the corresponding internal variables and algorithmic consistent tangents, with the coupling between these different components obtained through the imposition of the equilibrium (equal stress) among them. It is significant to observe that the consistent linearization of the discrete equations can be obtained not only in closed form, but also in a very modular form combining the different algorithmic consistent tangents associated to each component of the material's 
response, namely the elastoplastic and damage components. The representative numerical simulations presented herein illustrate the numerical performance of the proposed numerical algorithms.

We believe that the aforementioned advantages provided by the considered framework do lead to an improvement in the modeling and numerical simulation of continuum damage models. The simple models considered herein are only representative examples. We plan to explore further this framework. For example, the study of strain localization and its regularization is of our main interest. The analysis of failure indicators in elastoplastic damage models (namely, the singularity of tangent operators and associated acoustic tensors) can be found in the work of Doghri and Billardon (1995), Comi et al. (1995), and Rizzi et al. (1996), among others. The approach considered in this work leads to alternative forms of the associated tangent operators (as given by Eq. (3.10)), deserving a complete analysis along these lines. Similarly, the extension of the considered formulations, including the numerical algorithms presented in this paper to the finite deformation range is the main focus of our current work in this area.

\section{Acknowledgements}

We are indebted to H.L. Schreyer and N. Hansen for helpful comments and providing computer files with the experimental results presented in Section 2.1.1. Financial support for this research has been provided by the ONR under contract no. N00014-96-1-0818 with UC Berkeley. This support is gratefully acknowledged. We also acknowledge the support of the NSF under contract no. CMS-9703000 with UC Berkeley.

\section{References}

Agelet de Saracibar, C., Onate, E., 1989. Plasticity models for porous metals. In: Owen, D.R.J., Onate, E., Hinton, E. (Eds.), Proceedings of COMPLAS II, vol. 1. CIMNE, Barcelona, 139-151.

Armero, F., Oller, S., 1999. A general framework for continuum damage models. I. Infinitesimal models in stress space. Int. J. Solids Struct. 37, 7409-7436.

Ashby, M.F., Tomkins, B., 1980. In: Smith, Miller. (Eds.), Proceedings of ICM-3, vol. 1. p. 47.

Atkins, A.G., Mai, Y.W., 1985. Elastic and Plastic Fracture. Wiley, New York.

Bazant, Z., Oh, J., 1983. Crack band theory for fracture of concrete. Mater. Struct. RILEM 16, 155-177.

Chow, C.L., Wang, J., 1987. An anisotropic theory of elasticity for continuum damage mechanics. Int. J. Fract. 33, 3-16.

Chaboche, J.L., 1995. A continuum damage theory with anisotropic and unilateral damage. La Recherche Aerospatiale 2, $139-147$.

Comi, C., Berthaud, Y., Billardon, R., 1995. On localization in ductile-brittle materials under compressive loadings. Eur. J. Mech. A/Solids 14, 19-43.

Cordebois, J., Sidoroff, F., 1979. Damage induced elastic anisotropy. Colloque Euromech 115, Villard de Lans. In: Boehler, J.P. (Ed.), 1983. Mechanical Behavior of Anisotropic Solids. Nijhoff (Martinus), The Hague, pp. 761-774.

Doghri, I., Billardon, R., 1995. Investigation of localization due to damage in elastoplastic materials. Mech. Mater. 19, $129-149$.

Gurson, A.L., 1975. Plastic flow and fracture behavior of ductile materials incorporating void nucleation, growth and interaction. Ph.D. Dissertation, Brown University.

Hansen, N.R., Schreyer, H.L., 1994. A thermodynamically consistent framework for theories of elastoplasticity coupled with damage. Int. J. Solids Struct. 31, 359-389.

Hansen, N.R., Schreyer, H.L., 1995. Damage deactivation. ASME J. Appl. Mech. 62, 450-458.

Lemaitre, J., 1983. A continuous damage mechanics model for ductile failure. J. Engng. Mater. Tech. ASME 107, 83-89.

Luenberger, D.G., 1984. Linear and Nonlinear Programming. Addison-Wesley, Reading.

Needleman, A., Rice, J.R., 1978. Limits to the ductility set by plastic flow localization. In: Koistinen, D.P., Wang, N.M. (Eds.), Mechanics of Sheet Metal Forming. Plenum Press, New York.

Needleman, A., Tvergaard, V., 1984. An analysis of ductile rupture in notched bars. J. Mech. Phys. Solids 32, 461.

Ortiz, M., 1985. A constitutive theory for the inelastic behavior of concrete. Mech. Mater. 4, 67-93.

Rizzi, E., Maier, G., Willam, K., 1996. On failure indicators in multi-dissipative materials. Int. J. Solids Struct. 33, $20-22$.

Rots, J.G., Nauta, P., Kusters, G., Blaauwendraa, T., 1985. Smeared crack approach and fracture localization in concrete. Heron 30. 
Shima, S., Oyane, M., 1976. Plasticity theory for porous metals. Int. J. Mech. Sci. 18, 285-291.

Simo, J.C., Hughes, T.J.R., 1997. Plasticity and Viscoplasticity, Formulation and Numerical Analysis. Springer, Berlin preprint. Simo, J.C., Rifai, S., 1990. A class of mixed assumed strain methods and the method of incompatible modes. Int. J. Num. Meth. Engng. 29, 1595-1638.

Tvergaard, V., Needleman, A., 1984. Finite element analysis of localization plasticity. In: Oden, J.T., Carey, G.F. (Eds.), Finite Elements, vol. V. Prentice Hall, Englewood Cliffs. 